TRANSACTIONS OF THE

AMERICAN MATHEMATICAL SOCIETY

Volume 353, Number 6, Pages 2391-2426

S 0002-9947(00)02649-0

Article electronically published on July 18, 2000

\title{
ON THE TELESCOPIC HOMOTOPY THEORY OF SPACES
}

\author{
A. K. BOUSFIELD
}

\begin{abstract}
In telescopic homotopy theory, a space or spectrum $X$ is approximated by a tower of localizations $L_{n}^{f} X, n \geq 0$, taking account of $v_{n}$-periodic homotopy groups for progressively higher $n$. For each $n \geq 1$, we construct a telescopic Kuhn functor $\Phi_{n}$ carrying a space to a spectrum with the same $v_{n}$-periodic homotopy groups, and we construct a new functor $\Theta_{n}$ left adjoint to $\Phi_{n}$. Using these functors, we show that the $n$th stable monocular homotopy category (comprising the $n$th fibers of stable telescopic towers) embeds as a retract of the $n$th unstable monocular homotopy category in two ways: one giving infinite loop spaces and the other giving "infinite $L_{n}^{f}$-suspension spaces." We deduce that Ravenel's stable telescope conjectures are equivalent to unstable telescope conjectures. In particular, we show that the failure of Ravenel's $n$th stable telescope conjecture implies the existence of highly connected infinite loop spaces with trivial Johnson-Wilson $E(n)_{*}$-homology but nontrivial $v_{n}$-periodic homotopy groups, showing a fundamental difference between the unstable chromatic and telescopic theories. As a stable chromatic application, we show that each spectrum is $K(n)$-equivalent to a suspension spectrum. As an unstable chromatic application, we determine the $E(n)_{*}$-localizations and $K(n)_{*}$-localizations of infinite loop spaces in terms of $E(n)_{*}$-localizations of spectra under suitable conditions. We also determine the $E(n)_{*}$-localizations and $K(n)_{*}$-localizations of arbitrary Postnikov $H$-spaces.
\end{abstract}

\section{INTRODUCTION}

With the probable failure of Ravenel's telescope conjecture, a "telescopic" or "finite chromatic" approach to stable homotopy theory has emerged in its own right (see [Mil2, [Rav4], $\mathrm{MS}]$ ). In this approach, a spectrum $E$ is approximated at a prime $p$ by a tower $\left\{L_{n}^{f} E\right\}_{n>0}$ of localizations away from the finite $p$-local spectra of type $n+1$. Recall that a finite $p$-local spectrum $W$ is of type $n$ when $K(i)_{*} W=0$ for $i<n$ and $K(n)_{*} W \neq 0$, where $K(n)$ is the $n$th Morava $K$-theory. By results of Mitchell, Hopkins, and Smith, such a $W$ exists for each $n$ and admits a self-map $\omega: \Sigma^{d} W \rightarrow W$ with $K(n)_{*} \omega$ an isomorphism and with $K(i)_{*} \omega=0$ for $i \neq n$. Such an $\omega$ is called a $v_{n}$-map and leads to the $v_{n}$-periodic homotopy groups $v_{n}^{-1} \pi_{*}(E ; W)=\omega^{-1}[W, E]_{*}$ of a spectrum $E$. The localization $E \rightarrow L_{n}^{f} E$ preserves the $v_{i}$-periodic homotopy groups of $E$ for $i \leq n$ while annihilating those for $i>n$, and the fiber $M_{n}^{f} E$ of $L_{n}^{f} E \rightarrow L_{n-1}^{f} E$ captures the $v_{n}$-periodic homotopy groups alone. From this perspective, $\left\{L_{n}^{f} E\right\}_{n \geq 0}$ resembles a Postnikov tower. As suggested by Ravenel, we call the spectrum $M_{n}^{f} E$ monocular.

Received by the editors March 29, 1999.

2000 Mathematics Subject Classification. Primary 55P60; Secondary 55N20, 55P42, 55P65, $55 \mathrm{U} 35$.

Research partially supported by the National Science Foundation. 
For spaces, a similar telescopic theory is obtained using the nullification or periodization functors of [Bou7], Bou8], or [Dro. A pointed space $X$ is approximated at a prime $p$ by a tower $\left\{L_{n}^{f} X\right\}_{n \geq 0}$ of nullifications away from desuspensions of finite $p$-local spectra of type $n+1$. Like its stable counterpart, the nullification $X \rightarrow L_{n}^{f} X$ preserves the $v_{i}$-periodic homotopy groups of $X$ for $i \leq n$ while annihilating those for $i>n$, and the fiber $M_{n}^{f} X$ of $L_{n}^{f} X \rightarrow L_{n-1}^{f} X$ captures the $v_{n}$-periodic homotopy groups alone. We likewise call the space $M_{n}^{f} X$ monocular.

For $n \geq 1$, Kuhn Kuh2] introduced a remarkable functor $\phi_{n}$ from pointed spaces to spectra with $\phi_{n} \Omega^{\infty} E \simeq L_{K(n)} E$ for a spectrum $E$, where $L_{K(n)}$ is the $K(n)_{*^{-}}$ localization functor. This greatly extended a similar result of [Bou6] for $n=1$. Using Kuhn's basic approach, we now construct a telescopic Kuhn functor $\Phi_{n}$ from pointed spaces to spectra with $\Phi_{n} \Omega^{\infty} E \simeq \widehat{M}_{n}^{f} E$ and $v_{n}^{-1} \pi_{*}\left(\Phi_{n} X ; W\right) \cong$ $v_{n}^{-1} \pi_{*}(X ; W)$ (by Theorem 5.3) for a spectrum $E$ and pointed space $X$, where $\widehat{M}_{n}^{f}$ is a variant of the $n$th monocular functor $M_{n}^{f}$. Our key result (Theorem 5.4) provides a new functor $\Theta_{n}$ from spectra to pointed spaces which is homotopically left adjoint to $\Phi_{n}$ in the sense that

$$
\operatorname{map}_{*}\left(\Theta_{n} E, L_{n}^{f} X\right) \simeq \operatorname{map}_{*}\left(E, \Phi_{n} X\right) .
$$

In particular, $\widetilde{K(n)^{*}}\left(\Theta_{n} E\right) \simeq K(n)^{*}(E)$, so that $\Theta_{n} E$ behaves somewhat like an infinite desuspension of $E$. Moreover, we establish a natural equivalence $L_{n}^{f} \Sigma^{\infty} \Theta_{n} E \simeq$ $M_{n}^{f} E$ showing that monocular spectra are closely related to suspension spectra. This is carried by $L_{K(n)}$ to a natural equivalence $L_{K(n)} \Sigma^{\infty} \Theta_{n} E \simeq L_{K(n)} E$ showing that every spectrum is $K(n)$-equivalent to a suspension spectrum (see Corollary $5.8)$.

For $n \geq 1$, we let $\mathcal{M}_{n}^{f}$ be the $n$th stable monocular homotopy category comprising all $M_{n}^{f} E$ for spectra $E$, and we let $\mathcal{U} \mathcal{N}_{n}^{f}$ be the $n$th unstable monocular homotopy category comprising all $\left(M_{n}^{f} X\right)\left\langle d_{n}\right\rangle$ for spaces $X$, where $d_{n}$ is a certain integer increasing with $n$ and $\left\langle d_{n}\right\rangle$ denotes a $d_{n}$-connected cover. In Theorem 5.14, we deduce that $\mathcal{M}_{n}^{f}$ embeds as a retract of $\mathcal{U} \mathcal{N}_{n}^{f}$ in two ways given by the adjoint factorizations

$$
\begin{gathered}
\mathcal{M}_{n}^{f} \stackrel{\Theta_{n}}{\longleftrightarrow} \mathcal{U} \mathcal{N}_{n}^{f} \stackrel{L_{n}^{f} \Sigma^{\infty}}{\longrightarrow} \mathcal{M}_{n}^{f} \\
\mathcal{M}_{n}^{f} \stackrel{M_{n}^{f} \Phi_{n}}{\longleftarrow} \mathcal{U} \mathcal{N}_{n}^{f} \stackrel{\widetilde{\Omega}^{\infty}}{\longleftarrow} \mathcal{M}_{n}^{f}
\end{gathered}
$$

of the identity functor for $\mathcal{M}_{n}^{f}$, where $\widetilde{\Omega}^{\infty} E=\left(\Omega^{\infty} E\right)\left\langle d_{n}\right\rangle$. We note that the $\widetilde{\Omega}^{\infty}$-embedding of $\mathcal{M}_{n}^{f}$ in $\mathcal{U} \mathcal{N}_{n}^{f}$ consists of infinite loop spaces, while the $\Theta_{n^{-}}$ embedding of $\mathcal{M}_{n}^{f}$ in $\mathcal{U} \mathcal{N}_{n}^{f}$ consists of "infinite $L_{n}^{f}$-suspension spaces" since $\Theta_{n} E \simeq$ $L_{n}^{f} \Sigma^{k}\left(\Theta_{n} \Sigma^{-k} E\right)$ for $k \geq 0$. Moreover, there is a correspondence of unstable and stable maps given by isomorphisms $\left[\Theta_{n} E, \widetilde{\Omega}^{\infty} G\right] \cong[E, G]$ for $E, G \in \mathcal{M}_{n}^{f}$.

Ravenel's original telescope conjecture involves the mapping telescopes of $v_{n}$ maps, but may be reformulated to assert that each $K(n)_{*}$-equivalence of spectra is a $v_{n}$-periodic homotopy equivalence (see e.g. [Rav1], MS], or 6.13). Since the converse is true, this would give a perfect Whitehead theorem for $K(n)_{*}$-equivalences of spectra. From another standpoint, the telescope conjecture concerns the natural telescopic-to-chromatic transformation $L_{n}^{f} \rightarrow L_{n}$ where $L_{n}$ is the $E(n)_{*}$-localization functor. In fact, $L_{n}^{f} E \rightarrow L_{n} E$ is an equivalence for each spectrum $E$ if and only if the $m$ th telescope conjecture holds for each $m \leq n$. The $n$th telescope conjecture was proved for $n=1$ by Mahowald Mah and Miller Mil1, but has been strongly 
doubted for $n \geq 2$ since 1990 when Ravenel [Rav2] sketched a refutation for $n=2$ and $p \geq 5$. However, a completely convincing refutation has been extraordinarily hard to achieve, and Ravenel cautions us against drawing conclusions at this time.

To formulate an unstable telescope conjecture, we call a map $X \rightarrow Y$ of pointed spaces a virtual $K(n)_{*}$-equivalence when it induces a $K(n)_{*}$-equivalence $(\Omega X)\langle n+2\rangle \rightarrow(\Omega Y)\langle n+2\rangle$. By [Bou9], the virtual $K(n)_{*}$-equivalences behave much like periodic homotopy equivalences; for instance, in a ladder of fiber sequences

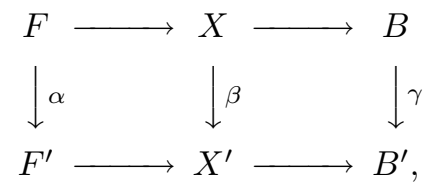

if any two of $\alpha, \beta$, and $\gamma$ are virtual $K(n)_{*}$-equivalences, then so is the third. Our unstable telescope conjecture asserts that each virtual $K(n)_{*}$-equivalence of pointed spaces is a $v_{n}$-periodic homotopy equivalence, and we prove that this is equivalent to the stable telescope conjecture for $K(n)_{*}$ (see Theorem 6.5). If the conjecture fails, then we can deduce the existence of highly connected infinite loop spaces with trivial $E(n)_{*}$-homology but nontrivial $v_{n}$-periodic homotopy groups (see Theorem $6.11)$.

Our telescopic results have important chromatic applications. They enable us to determine the $E(n)_{*}$-localizations of many infinite loop spaces in terms of the $E(n)_{*}$-localizations of spectra, very much as we determined the $E(1)_{*}$-localizations of infinite loop spaces in Bou4]. For a connected infinite loop space $\Omega_{0}^{\infty} E$, we show that $L_{n} \Omega_{0}^{\infty} E$ is given by $\Omega_{0}^{\infty} L_{n} E$ with low dimensional adjustments under suitable conditions (Theorem 8.1). These conditions hold, for instance, when $n \leq 2$ or when $E$ is a $B P$-module spectrum. The $K(n)_{*}$-localizations of spaces are very closely related to $E(n)_{*}$-localizations; if $Y$ is a connected $H$-space or simply connected space, then $L_{K(n)} Y \simeq\left(L_{n} Y\right)_{p}^{\wedge}$ (see Theorem 7.3). In particular, $L_{K(n)} \Omega_{0}^{\infty} E$ is given by $\Omega_{0}^{\infty}\left(L_{n} E\right)_{p}^{\wedge}$ with low dimensional adjustments under suitable conditions (see Theorem 8.2), and may differ substantially from $\Omega_{0}^{\infty} L_{K(n)} E$. We also determine $L_{n} Y$ and $L_{K(n)} Y$ for any Postnikov $H$-space $Y$ using work of Hopkins, Ravenel, and Wilson HRW].

Much of our effort is devoted to constructing the functors $\Phi_{n}$ and $\Theta_{n}$ using the technology of model categories. We give a much improved version of the BousfieldFriedlander $[\mathrm{BF}]$ construction of new model categories from old ones with " $Q$ structures," and deduce that each nullification functor determines a proper model category for spaces (see Theorem 9.9). In particular, the telescopic functor $L_{n}^{f}$ determines a proper model category structure for spaces, giving the $n$th telescopic homotopy category. We construct a chain of right Quillen functors and left Quillen equivalences from this model category to the model category of spectra, and we compose their total derived functors to define $\Phi_{n}$. We then construct an adjoint chain of functors and compose their total derived functors to define $\Theta_{n}$. The homotopy functors $\Phi_{n}$ and $\Theta_{n}$ can be induced by simplicial functors between the categories of spaces and spectra.

The paper is organized as follows. In Section 2 we discuss the general theory of relative homological localizations of spectra, and in Section 3 we review the telescopic homotopy theory of spectra. Although this material is more or less known, it is needed to establish notation, terminology, and background. In Section 
4 we introduce the telescopic homotopy theory of spaces. In Section 5 we state our main theorems on the existence and basic properties of the functors $\Phi_{n}$ and $\Theta_{n}$, and we derive our embedding theorem for monocular homotopy categories. In Section 6 we prove the equivalence of the stable and unstable telescope conjectures. In Section 7 we determine the $E(n)_{*}$-localizations and $K(n)_{*}$-localizations of Postnikov $H$ spaces. In Section 8 we determine the $E(n)_{*}$-localizations and $K(n)_{*}$-localizations of infinite loop spaces under suitable conditions. In Section 9 we show that a nullification functor, such as $L_{n}^{f}$, determines a proper model category structure for spaces. In Sections 10-13 we construct the functors $\Phi_{n}$ and $\Theta_{n}$ and establish their basic properties listed in Theorems 5.3 and 5.4.

We work simplicially so that a "space" is a simplicial set, and a "spectrum" is as in $[\mathrm{BF}$ or [HSS] 4.2]. However, to make the presentation more accessible, we frequently work in the homotopy categories $H o_{*}$ of pointed spaces and $\mathrm{Ho}^{s}$ of spectra. We develop our results in telescopic and chromatic homotopy theory at a fixed but arbitrary prime $p$.

We are very grateful to the referee for critical comments and suggestions which have helped to make this work more accessible.

\section{Relative homological LOCALizATions of SPECTRA}

To set the stage for our discussion of telescopic stable homotopy theory in Section 3, we shall establish an equivalence between two sorts of relative homological localizations of spectra. We work in the stable homotopy category of spectra and assume familiarity with

2.1. Homological localizations and acyclizations of spectra. For a spectrum $E$, we let $X \rightarrow L_{E} X$ and $C_{E} X \rightarrow X$ denote the $E_{*}$-localization and $E_{*}$-acyclization of a spectrum $X$, and we obtain a homotopy cofiber sequence $C_{E} X \rightarrow X \rightarrow$ $L_{E} X$ (see [Bou2], Rav1]). We let $\mathcal{L}_{E} \subset H o^{s}$ (resp. $\left.\mathcal{C}_{E} \subset H o^{s}\right)$ denote the full subcategory of $E_{*}$-local (resp. $E_{*}$-acyclic) spectra.

2.2. Ayclicity classes of spectra. The functors $L_{E}$ and $C_{E}$ depend only on the equivalence class $\langle E\rangle$ obtained by considering two spectra as equivalent when their homology theories have the same acyclic spectra. These acyclicity classes $\langle E\rangle$ are studied at length in Bou1, Rav1, and [HP]. They are partially ordered, where $\langle G\rangle \geq\langle E\rangle$ means that each $G_{*}$-acyclic spectrum is $E_{*}$-acyclic, and they have the usual smash and wedge operations. A class $\langle X\rangle$ may have a complement $\langle X\rangle^{c}$, which is uniquely determined by the conditions $\langle X\rangle \wedge\langle X\rangle^{c}=\langle 0\rangle$ and $\langle X\rangle \vee\langle X\rangle^{c}=$ $\langle S\rangle$ where $S$ is the sphere spectrum. For a class $\langle W\rangle$ and a complemented class $\langle X\rangle$, the difference class $\langle W\rangle \backslash\langle X\rangle$ is defined as $\langle W\rangle \wedge\langle X\rangle^{c}$.

2.3. Relative homological localizations. For spectra $E$ and $G$ with $\langle G\rangle \geq\langle E\rangle$, there is a natural map $L_{G} X \rightarrow L_{E} X$ with homotopy fiber $C_{E} L_{G} X$ for a spectrum $X$. We regard $C_{E} L_{G} X$ as a relative homological localization of $X$ giving its $G_{*}$-local $E_{*}$-acyclic part. When $\langle E\rangle$ is complemented, we may choose a representative $G \backslash E$ of $\langle G\rangle \backslash\langle E\rangle$ and regard $L_{G \backslash E} X$ as another sort of relative homological localization of $X$. We shall show that $C_{E} L_{G}$ and $L_{G \backslash E}$ are functorially equivalent when $E$ is smashing. 
2.4. Smashing spectra. As in Rav1, a spectrum $E$ is called smashing when the natural map $X \wedge L_{E} S \rightarrow L_{E} X$ is an equivalence for all spectra $X$. When $E$ is smashing, $X \wedge C_{E} S \rightarrow C_{E} X$ is also an equivalence, and $\langle E\rangle$ is complemented with $\langle E\rangle=\left\langle L_{E} S\right\rangle$ and $\langle E\rangle^{c}=\left\langle C_{E} S\right\rangle$.

Theorem 2.5. For a smashing spectrum $E$ and spectrum $G$ with $\langle G\rangle \geq\langle E\rangle$, the relative homological localization functors $C_{E} L_{G}$ and $L_{G \backslash E}$ are related by natural equivalences $L_{G \backslash E}\left(C_{E} L_{G} X\right) \simeq L_{G \backslash E} X$ and $C_{E} L_{G}\left(L_{G \backslash E} X\right) \simeq C_{E} L_{G} X$ for $X \in$ $H o^{s}$. Moreover, they restrict to adjoint functors $C_{E} L_{G}: H o^{s} \rightleftarrows \mathcal{L}_{G}: L_{G \backslash E}$ which in turn restrict to adjoint equivalences $C_{E} L_{G}: \mathcal{L}_{G \backslash E} \rightleftarrows \mathcal{C}_{E} \wedge \mathcal{L}_{G}: L_{G \backslash E}$. Furthermore, $L_{G \backslash E} X \simeq F\left(C_{E} S, L_{G} X\right) \simeq L_{S \backslash E} L_{G} X$ for $X \in H o^{s}$.

Cases of this result have been proved in Bou3], Bou8], [HPS], and [HSt]. Our proof in 2.7 will use the following categorical lemma. For a class $\mathcal{W}$ of maps in a category $\mathcal{A}$, an object $J \in \mathcal{A}$ is called $\mathcal{W}$-local (resp. $\mathcal{W}$-colocal) when $f^{*}$ : $\operatorname{Hom}(B, J) \cong \operatorname{Hom}(A, J)$ (resp. $\left.f^{*}: \operatorname{Hom}(J, A) \cong \operatorname{Hom}(J, B)\right)$ for each map $f$ : $A \rightarrow B$ in $\mathcal{W}$. A $\mathcal{W}$-localization of $A \in \mathcal{A}$ consists of a map $A \rightarrow I$ in $\mathcal{W}$ such that $I$ is $\mathcal{W}$-local, and a $\mathcal{W}$-colocalization consists of a map $J \rightarrow A$ in $\mathcal{W}$ such that $J$ is $\mathcal{W}$-colocal.

Lemma 2.6. If each object of $\mathcal{A}$ has both a $\mathcal{W}$-localization and a $\mathcal{W}$-colocalization, then:

(i) the $\mathcal{W}$-colocalization functor $C: \mathcal{A} \rightarrow \mathcal{A}$ is left adjoint to the $\mathcal{W}$-localization functor $L: \mathcal{A} \rightarrow \mathcal{A}$;

(ii) there are natural isomorphisms $L C X \cong L X$ and $C L X \cong C X$ for $X \in \mathcal{A}$;

(iii) $C$ and $L$ restrict to adjoint equivalences between the full subcategories of $\mathcal{W}$-local objects and of $\mathcal{W}$-colocal objects in $\mathcal{A}$.

Proof. $C$ is a left adjoint to $L$ via the natural bijections

$$
\operatorname{Hom}(C X, Y) \cong \operatorname{Hom}(C X, L Y) \cong \operatorname{Hom}(X, L X) .
$$

The lemma follows easily since $C$ and $L$ carry maps of $\mathcal{W}$ to isomorphisms by adjunction arguments.

2.7. Proof of Theorem 2.5. Let $\mathcal{W}$ be the class of $C_{E}$-equivalences in $H o^{s}$. Then each spectrum $X$ has a $\mathcal{W}$-localization $X \rightarrow L_{S \backslash E} X$ and a $\mathcal{W}$-colocalization $C_{E} X \rightarrow X$. Thus $L_{S \backslash E}: H o^{s} \rightarrow H o^{s}$ is right adjoint to $C_{E}: H o^{s} \rightarrow H o^{s}$ by Lemma 2.6, and hence $L_{S \backslash E} X \simeq F\left(C_{E} S, X\right)$ for $X \in H o^{s}$ since $C_{E} X \simeq X \wedge C_{E} S$. If $W \in H o^{s}$ is $G \backslash E_{*}$-acyclic, then $W \wedge\left(G \wedge C_{E} S\right) \simeq 0$ and thus $\left[W, F\left(C_{E} S, L_{G} X\right)\right]=0$ for $X \in H o^{s}$. Hence $F\left(C_{E} S, L_{G} X\right)$ is $G \backslash E_{*}$-local and $L_{G \backslash E} X \simeq F\left(C_{E} S, L_{G} X\right) \simeq$ $L_{S \backslash E} L_{G} X$. The theorem now follows easily.

Examples of smashing spectra arise from the theory of

2.8. Homotopical trivializations and cotrivialization of spectra. As in Bou1 for $W \in H o^{s}$, we say that a spectrum $I$ is $W$-trivial when $[W, I]_{*}=0$ and say that a spectrum $J$ is $W$-cotrivial when $[J, Y]_{*}=0$ for each $W$-trivial spectrum $Y$. By [Bou1, 1.7], each spectrum $X$ has a $W$-trivialization $X \rightarrow L^{W} X$ and a $W$-cotrivialization $C^{W} X \rightarrow X$, and we obtain a homotopy (co)fiber sequence 
$C^{W} X \rightarrow X \rightarrow L^{W} X$. We let $\mathcal{L}^{W} \subset H o^{s}$ (resp. $\mathcal{C}^{W} \subset H o^{s}$ ) denote the full subcategory of $W$-trivial (resp. $W$-cotrivial) spectra. By [Bou1, 1.6], $\mathcal{C}^{W}$ is the smallest full subcategory of $H o^{s}$ which contains $W$, is closed under arbitrary wedges, and contains all three spectra in a cofiber sequence when it contains any two. We remark that homological localizations of spectra are actually examples of homotopical trivializations by [Bou2, 1.14].

The following theorem generalizes a result of [Bou2, 3.5] or [Mil2] showing that the spectrum $L^{W} S$ is smashing when $W$ is a wedge of finite spectra.

Theorem 2.9. Let $W$ be a spectrum such that $\mathcal{L}^{W}$ is closed under arbitrary wedges. Then $L^{W} S$ is smashing with $\left\langle L^{W} S\right\rangle=\langle W\rangle^{c}$, and the $L^{W} S_{*}$-localization of a spectrum $X$ is given by the $W$-trivialization $X \rightarrow L^{W} X$.

Proof. If $E \in \mathcal{L}^{W}$, then $X \wedge E \in \mathcal{L}^{W}$ for each $X \in H o^{s}$, since $\mathcal{C}^{E} \subset \mathcal{L}^{W}$ by 2.8 . Using the cofiber sequence $X \wedge C^{W} S \rightarrow X \wedge S \rightarrow X \wedge L^{W} S$ with $X \wedge C^{W} S \in \mathcal{C}^{W}$ and $X \wedge L^{W} S \in \mathcal{L}^{W}$, we obtain natural equivalences $X \wedge C^{W} S \simeq C^{W} X$ and $X \wedge L^{W} S \simeq L^{W} X$ for $X \in H o^{s}$. Since $W \wedge C^{W} S \simeq W$ and $C^{W} S \wedge L^{W} S \simeq 0$, we easily deduce $\langle W\rangle=\left\langle C^{W} S\right\rangle$ and $\langle W\rangle^{c}=\left\langle L^{W} S\right\rangle$ as required.

\section{The TELESCOPiC HOMOTOPY THEORY OF SPECTRA}

We now discuss the "finite chromatic" or "telescopic" approach to stable homotopy theory in which spectra are approximated by their homotopical trivializations away from finite spectra of progressively higher types as in Mil2, Rav4], or [MS. Using our knowledge of relative homological localizations, we establish an equivalence between two sorts of "monocular" stable homotopy categories. Let $p$ be a fixed prime, and let $K(n)$ be the $n$th Morava $K$-theory spectrum at $p$ for $n \geq 0$ where $K(0)=H Q$.

3.1. Types of spectra. The type of a finite $p$-local spectrum $W$ is the smallest integer $n \geq 0$ such that $K(n)_{*} W \neq 0$, or is $\infty$ when there is no such $n$ (i.e., when $W \simeq 0$ ). A spectrum $W$ of type $n$ exists for each $n \geq 0$, and any two such spectra have the same acyclicity class; moreover, this class decreases monotonely as $n$ increases.

3.2. Telescopic towers of spectra. For $n \geq-1$, let $F(n+1)$ be a finite $p$-local spectrum of type $n+1$, let $\check{F}(n+1)=F(n+1) \vee \bigvee_{q \neq p} S / q$ be the wedge of $F(n+1)$

with the Moore spectra $S / q$ for all primes $q \neq p$, and let $S(n)$ be the $\check{F}(n+1)$ trivialization of the sphere spectrum $S$. We note that $S(n)$ does not depend on the choice of $F(n+1)$, and we have $S(-1)=0$ and $S(0)=H Q$. By Theorem 2.9, $S(n)$ is smashing with $\langle S(n)\rangle=\langle\check{F}(n+1)\rangle^{c}$ and hence with $\langle S(m)\rangle \leq\langle S(n)\rangle$ for $m \leq n$. For a spectrum $X$, we write $L_{n}^{f} X=L_{S(n)} X$ and $C_{n}^{f} X=C_{S(n)} X$ and obtain the natural telescopic tower

$$
L_{0}^{f} X \leftarrow L_{1}^{f} X \leftarrow \cdots \leftarrow L_{n-1}^{f} X \leftarrow L_{n}^{f} X \leftarrow \cdots
$$

under $X$. The homotopy fiber $M_{n}^{f} X$ of $L_{n}^{f} X \rightarrow L_{n-1}^{f} X$ is given by $M_{n}^{f} X=$ $C_{n-1}^{f} L_{n}^{f} X$ and is called the $n$th monocular part of $X$. It has a variant $\widehat{M}_{n}^{f} X=$ $L_{T(n)} X$ where $T(n)=S(n) \wedge F(n)$ is the "difference spectrum" with

$$
\langle T(n)\rangle=\langle S(n)\rangle \backslash\langle S(n-1)\rangle=\langle F(n)\rangle \backslash\langle F(n+1)\rangle .
$$


We let $\mathcal{L}_{n}^{f} \subset H o^{s}, \mathcal{M}_{n}^{f} \subset H o^{s}$, and $\widehat{\mathcal{M}}_{n}^{f} \subset H o^{s}$ respectively denote the full subcategories given by all $L_{n}^{f} X, M_{n}^{f} X$, and $\widehat{M}_{n}^{f} X$ for $X \in H o^{s}$. By Theorem 2.5 we have

Theorem 3.3. For $n \geq 0$, the functors $M_{n}^{f}$ and $\widehat{M}_{n}^{f}$ are related by natural equivalences $\widehat{M}_{n}^{f} M_{n}^{f} X \simeq \widehat{M}_{n}^{f} X$ and $M_{n}^{f} \widehat{M}_{n}^{f} X \simeq M_{n}^{f} X$ for $X \in H o^{s}$. Moreover, they restrict to adjoint functors $M_{n}^{f}: H o^{s} \rightleftarrows \mathcal{L}_{n}^{f}: \widehat{M}_{n}^{f}$ which in turn restrict to adjoint equivalences $M_{n}^{f}: \widehat{\mathcal{M}}_{n}^{f} \rightleftarrows \mathcal{M}_{n}^{f}: \widehat{M}_{n}^{f}$. Furthermore, $\widehat{M}_{n}^{f} X \simeq F\left(C_{n-1}^{f} S, L_{n}^{f} X\right) \simeq$ $F\left(C_{n-1}^{f} S_{(p)}, L_{n}^{f} X\right) \simeq L_{\check{F}(n)} L_{n}^{f} X \simeq L_{F(n)} L_{n}^{f} X$ for $X \in H o^{s}$.

A similar result is proved in [HSt, Theorem 6.19], for the monochromatic functors $M_{n}$ and $\widehat{M}_{n}$, and can also be derived from Theorem 2.5. We immediately deduce

Corollary 3.4. For spectra $X, Y \in H o^{s}$ and $n>0$, there are isomorphisms

$$
\left[M_{n}^{f} X, M_{n}^{f} Y\right] \cong\left[M_{n}^{f} X, \widehat{M}_{n}^{f} Y\right] \cong\left[\widehat{M}_{n}^{f} X, \widehat{M}_{n}^{f} Y\right] .
$$

The functors $L_{n}^{f}, M_{n}^{f}$, and $\widehat{M}_{n}^{f}$ are perhaps best understood in terms of

3.5. $v_{n}$-periodic homotopy groups. Let $\mathcal{F}_{n} \subset H o^{s}$ be the full subcategory of finite $p$-local spectra of type $\geq n$. For a spectrum $W \in \mathcal{F}_{n}$ with $n>0$, a $v_{n}$-map is a map $\omega: \Sigma^{d} W \rightarrow W$ with $d>0$ such that $K(n)_{*} \omega$ is an isomorphism and $K(i)_{*} \omega=0$ for all $i \neq n$. By the periodicity theorem of Hopkins and Smith (see Hop, HSm, Rav3], each spectrum $W \in \mathcal{F}_{n}$ has a $v_{n}$-map, which is unique and natural up to iteration. The $v_{n}$-periodic homotopy groups of a spectrum $X \in H o^{s}$ with coefficients in a spectrum $W \in \mathcal{F}_{n}$ are defined by

$$
v_{n}^{-1} \pi_{*}(X ; W)=\omega^{-1}[W, X]_{*}=\operatorname{colim}_{i}\left[\Sigma^{i d} W, X\right]_{*}
$$

for $n>0$ using the sequence

$$
W \stackrel{\omega}{\longleftarrow} \Sigma^{d} W \stackrel{\omega}{\longleftarrow} \Sigma^{2 d} W \leftarrow \cdots
$$

for a $v_{n}$-map $\omega: \Sigma^{d} W \rightarrow W$, and by $v_{0}^{-1} \pi_{*}(X ; W)=\left[W, X_{Q}\right]_{*}$ for $n=0$. The periodicity theorem ensures that $v_{n}^{-1} \pi_{*}(X ; W)$ does not depend on the choice of $\omega$ and is functorial in $X$ and $W$. In fact, $v_{n}^{-1} \pi_{*}(-; W)$ is a homology theory by

Lemma 3.6. For a spectrum $X$ and $W \in \mathcal{F}_{n}$ with $n \geq 0$, there is a natural isomorphism $v_{n}^{-1} \pi_{*}(X ; W) \cong(S(n) \wedge D W)_{*} X$ where $D W=F\left(W, S_{(p)}\right)$ is the p-local Spanier-Whitehead dual of $W$.

Proof. We may assume $n>0$. Since $D \omega: D W \rightarrow \Sigma^{-d} D W$ is a $v_{n}$-map in $\mathcal{F}_{n}$, its homotopy cofiber is in $\mathcal{F}_{n+1}$, and the map $D \omega \wedge C$ is nilpotent for each $C \in \mathcal{F}_{n+1}$ by the nilpotence theorem of Devinatz, Hopkins, and Smith (see Hop], [HSm, [Rav3]). Hence, the sequence

$$
D W \stackrel{D \omega}{\longrightarrow} \Sigma^{-d} D W \stackrel{D \omega}{\longrightarrow} \Sigma^{-2 d} D W \stackrel{D \omega}{\longrightarrow} \cdots
$$

has the telescope $L_{n}^{f} D W \simeq S(n) \wedge D W$ and

$$
v_{n}^{-1} \pi_{*}(X ; W) \cong \operatorname{colim}_{i}\left(\Sigma^{-i d} D W\right)_{*} X \cong(S(n) \wedge D W)_{*} X .
$$


3.7. $v_{n}$-periodic homotopy equivalences. For a finite $p$-local spectrum $W$ of type $n$, we have $\langle S(n) \wedge D W\rangle=\langle S(n) \wedge F(n)\rangle=\langle T(n)\rangle$. Thus by Lemma 3.6, a $v_{n}^{-1} \pi_{*}(-; W)$-equivalence of spectra is the same as a $T(n)_{*}$-equivalence and does not depend on the choice of $W$. It will be called a $v_{n}^{-1} \pi_{*}$-equivalence. A map which is a $v_{i}^{-1} \pi_{*}$-equivalence for $0 \leq i \leq n$ will be called a $\bigoplus_{i=0}^{n} v_{i}^{-1} \pi_{*}$-equivalence and is the same as an $S(n)_{*}$-equivalence since $\langle S(n)\rangle=\langle T(0)\rangle \vee \cdots \vee\langle T(n)\rangle$.

3.8. An interpretation of the telescopic tower. For a spectrum $X$ and $n \geq 0$, we may interpret $L_{n}^{f} X$ as the $\bigoplus_{i=0}^{n} v_{i}^{-1} \pi_{*}$-localization of $X$, and we obtain

$$
\begin{array}{r}
v_{i}^{-1} \pi_{*}\left(L_{n}^{f} X ; F(i)\right) \cong \begin{cases}v_{i}^{-1} \pi_{*}(X ; F(i)) & \text { for } i \leq n, \\
0 & \text { for } i>n,\end{cases} \\
v_{i}^{-1} \pi_{*}\left(M_{n}^{f} X ; F(i)\right) \cong \begin{cases}v_{i}^{-1} \pi_{*}(X ; F(i)) & \text { for } i=n, \\
0 & \text { for } i \neq n,\end{cases}
\end{array}
$$

for $i \geq 0$ where $F(i)$ is a finite $p$-local spectrum of type $i$. Thus the telescopic tower $\left\{L_{n}^{f} X\right\}_{n \geq 0}$ resembles a Postnikov tower for $X$, while its monocular fibers $M_{n}^{f} X$ resemble Eilenberg-MacLane objects. We may likewise interpret $\widehat{M}_{n}^{f} X$ as the $v_{n}^{-1} \pi_{*}$-localization of $X$.

\section{The telescopic homotopy theory of SPACES}

We now introduce a "finite chromatic" or "telescopic" approach to the homotopy theory of spaces, using the theory of homotopical nullifications (= periodizations) developed by Dror-Farjoun [Dro] and the author [Bou7], Bou8]. For convenience, we work primarily in the homotopy category $\mathrm{Ho}_{*}$ of pointed spaces (i.e. pointed simplicial sets), and start by recalling

4.1. Nullifications and conullifications of spaces. For a space $A \in H o_{*}$, we say that a space $Y \in H o_{*}$ is $A$-null if the map $A \rightarrow *$ induces an equivalence $Y \simeq \operatorname{map}(A, Y)$ of unpointed mapping spaces. When $Y$ is connected, this means that the pointed mapping $\operatorname{space}^{\operatorname{map}_{*}}(A, Y)$ is contractible. We say that a space $J \in H o_{*}$ is $A$-conull if $\operatorname{map}_{*}(J, Y) \simeq *$ for each $A$-null space $Y \in H o_{*}$. By Bou7] or [Dro], each space $X \in H o_{*}$ has an $A$-nullification $X \rightarrow P_{A} X$ going universally to an $A$-null target, and has an $A$-conullification $\bar{P}_{A} X \rightarrow X$ coming universally from an $A$-conull source. These form a homotopy fiber sequence $\bar{P}_{A} X \rightarrow X \rightarrow P_{A} X$ by Dro, p. 37]. We note that the terms " $A$-null" and " $A$-nullification" have exactly the same meanings as the earlier terms " $A$-periodic" and " $A$-periodization" used in Bou7.

4.2. Nullity classes and relative nullifications. The functors $P_{A}$ and $\bar{P}_{A}$ depend only on the equivalence class $\langle A\rangle$ obtained by considering two spaces as equivalent when they have the same null spaces. These nullity classes $\langle A\rangle$ are studied in Bou7] and Dro. They are partially ordered where $\langle A\rangle \leq\langle B\rangle$ means that each $B$-null space is $A$-null, which happens if and only if $P_{B} A \simeq *$. (The opposite ordering is used in Dro.) If $\langle A\rangle \leq\langle B\rangle$, then for $X \in H o_{*}$ there is a natural map $P_{A} X \rightarrow P_{B} X$ with homotopy fiber $\bar{P}_{B} P_{A} X$, and we regard $\bar{P}_{B} P_{A} X$ as a relative nullification of $X$, giving its $A$-null $B$-conull part. 
4.3. Telescopic towers of spaces. For $n \geq 0$, we choose a suspension space $A(n+1) \in H o_{*}$ such that $\Sigma^{\infty} A(n+1)$ is a finite $p$-local spectrum of type $n+1$ with the connectivity of $\widetilde{H}_{*}(A(n+1) ; Z / p)$ as low as possible. For instance, we might choose $A(1)=M(Z / p, 2)=S^{2} \cup_{p} e^{3}$. We let $d_{n}-1$ denote the connectivity of $\widetilde{H}_{*}(A(n+1) ; Z / p)$ and observe that $d_{0}=2, d_{n+1} \geq d_{n}$, and $d_{n} \geq n+1$ by Bou7. 13.8]. We let

$$
\check{A}(n+1)=A(n+1) \vee \bigvee_{q \neq p} M(Z / q, 2)
$$

be the wedge of $A(n+1)$ with the $Z / q$-Moore spaces $M(Z / q, 2)$ for primes $q \neq p$. Then by Bou7, 9.15], the nullity class $\langle\check{A}(n+1)\rangle$ does not depend on the choice of $A(n+1)$, and it decreases monotonely as $n$ increases. For a pointed space $X$, we write $L_{n}^{f} X=P_{\check{A}(n+1)} X$ and $C_{n}^{f} X=\bar{P}_{\check{A}(n+1)} X$, and we obtain the natural telescopic tower

$$
L_{0}^{f} X \leftarrow L_{1}^{f} X \leftarrow \cdots \leftarrow L_{n-1}^{f} X \leftarrow L_{n}^{f} X \leftarrow \cdots
$$

under $X$. For $n \geq 1$, the homotopy fiber $M_{n}^{f} X$ of $L_{n}^{f} X \rightarrow L_{n-1}^{f} X$ is given by $M_{n}^{f} X=C_{n-1}^{f} L_{n}^{f} X$ and is called the $n$th monocular part of $X$. We let $\mathcal{U} \mathcal{L}_{n}^{f} \subset H o_{*}$ and $\mathcal{U} \mathcal{M}_{n}^{f} \subset H o_{*}$ respectively denote the full subcategories given by all $L_{n}^{f} X$ and $M_{n}^{f} X$ for $X \in H o_{*}$. As will be explained below in $4.11, L_{n}^{f} X$ is very closely related to the $v_{n}$-periodization $P_{v_{n}} X$ of [Bou7].

4.4. Simplicial functoriality. Since the functors $L_{n}^{f}: H o_{*} \rightarrow H o_{*}$ for $n \geq 0$ are nullifications, they are induced as in [Bou7, 2.10] by simplicial functors $L_{n}^{f}: \mathcal{S S}_{*} \rightarrow$ $\mathcal{S S}_{*}$ which may be constructed with simplicial natural transformations giving a functorial telescopic tower $X \rightarrow\left\{L_{n}^{f} X\right\}_{n \geq 0}$ for $X \in \mathcal{S S}_{*}$.

The telescopic tower is perhaps best understood in terms of

4.5. $v_{n}$-periodic homotopy groups. The $v_{n}$-periodic homotopy groups of a space $X \in H_{o_{*}}$ with coefficients in a spectrum $W \in \mathcal{F}_{n}$ are defined for $n \geq 1$ by

$$
v_{n}^{-1} \pi_{*}(X ; W)=\omega^{-1}[W, X]_{*}=\operatorname{colim}_{i}\left[\Sigma^{i d} W, X\right]_{*}
$$

using the eventual desuspension to $\mathrm{Ho}_{*}$ of the sequence

$$
W \stackrel{\omega}{\longleftarrow} \Sigma^{d} W \stackrel{\omega}{\longleftarrow} \Sigma^{2 d} W \longleftarrow \cdots
$$

for a $v_{n}$-map $\omega: \Sigma^{d} W \rightarrow W$. As in the stable case $(3.5), v_{n}^{-1} \pi_{*}(X ; W)$ does not depend on the choice of $\omega$ and is functorial in $X$ and $W$. A map $f: X \rightarrow Y$ in $H o_{*}$ will be called a $v_{n}^{-1} \pi_{*}$-equivalence when $f_{*}: v_{n}^{-1} \pi_{*}(X ; W) \cong v_{n}^{-1} \pi_{*}(Y ; W)$ for some (and hence all) $W \in \mathcal{F}_{n}$ of type $n$ (see [Bou7, 11.11]). It will be called a $v_{0}^{-1} \pi_{*}$-equivalence when $f_{*}: Q \otimes \pi_{*} X \cong Q \otimes \pi_{*} Y$ assuming that $X$ and $Y$ are nilpotent.

4.6. An interpretation of the telescopic tower. For a space $X \in H o_{*}$, we have

$$
\pi_{i} L_{0}^{f} X \cong \begin{cases}\pi_{i} X & \text { for } i<2 \\ \pi_{i} X / \tau \pi_{i} X & \text { for } i=2 \\ \pi_{i} X \otimes Q & \text { for } i>2\end{cases}
$$


by [Bou7, Theorem 5.2], where $\tau \pi_{i} X$ is the torsion subgroup of $\pi_{i} X$. For $n \geq 1$ and $i \geq 1$, we obtain

$$
\begin{array}{r}
v_{i}^{-1} \pi_{*}\left(L_{n}^{f} X ; F(i)\right) \cong \begin{cases}v_{i}^{-1} \pi_{*}(X ; F(i)) & \text { for } i \leq n, \\
0 & \text { for } i>n,\end{cases} \\
v_{i}^{-1} \pi_{*}\left(M_{n}^{f} X ; F(i)\right) \cong \begin{cases}v_{i}^{-1} \pi_{*}(X ; F(i)) & \text { for } i=n, \\
0 & \text { for } i \neq n\end{cases}
\end{array}
$$

by 4.11 below and [Bou7, 11.5] where $F(i)$ is a finite $p$-local spectrum of type $i$. Thus the telescopic tower $\left\{L_{n}^{f} X\right\}_{n \geq 0}$ resembles a Postnikov tower for $X$, capturing progressively higher types of periodic homotopy.

This interpretation can be strengthened for highly connected spaces. For $j \geq 0$ let $Y\langle j\rangle$ denote the $j$-connected cover of a space $Y \in H o_{*}$, and let $H o_{*}\langle j\rangle \subset H o_{*}$ denote the full subcategory of $j$-connected spaces. By 4.11 below and Theorems 11.5, 13.1, and 13.3 of [Bou7], we have

Theorem 4.7. For $n \geq 0$ and $X \in H o_{*}\left\langle d_{n}\right\rangle$, the map $X \rightarrow L_{n}^{f} X$ is $a \bigoplus_{i=0}^{n} v_{i}^{-1} \pi_{*}$ localization of $X$ in $H_{*}\left\langle d_{n}\right\rangle$. Moreover, for $Y \in H o_{*}$, there is a natural equivalence $L_{n}^{f}\left(Y\left\langle d_{n}\right\rangle\right) \simeq\left(L_{n}^{f} Y\right)\left\langle d_{n}\right\rangle$.

Corollary 4.8. For $n \geq 0$, a map in $H o_{*}\left\langle d_{n}\right\rangle$ is an $L_{n}^{f}$-equivalence if and only if it is $a \bigoplus_{i=0}^{n} v_{i}^{-1} \pi_{*}$-equivalence.

For $n \geq 1$, we write $N_{n}^{f} X=\left(M_{n}^{f} X\right)\left\langle d_{n}\right\rangle$ and let $\mathcal{U} \mathcal{N}_{n}^{f} \subset H o_{*}$ denote the full subcategory given by all $N_{n}^{f} X$ for $X \in H o_{*}$. We call $\mathcal{U} \mathcal{N}_{n}^{f}$ the $n$th monocular homotopy category of spaces.

Lemma 4.9. For $n \geq 1, \mathcal{U N}_{n}^{f}$ is contained in $\mathcal{U M}_{n}^{f}$.

Proof. For $X \in H o_{*}$, we have $L_{n-1}^{f}\left(N_{n}^{f} X\right) \simeq *$ by Corollary 4.8 and have $N_{n}^{f} X$ in $\mathcal{U} \mathcal{L}_{n}^{f}$ by Theorem 4.7 since $N_{n}^{f} X=\left(M_{n}^{f} X\right)\left\langle d_{n}\right\rangle$ with $M_{n}^{f} X \in \mathcal{U} \mathcal{L}_{n}^{f}$.

Thus, $\mathcal{U} \mathcal{N}_{n}^{f}$ is the full subcategory of $\mathcal{U} \mathcal{M}_{n}^{f}$ given by its $d_{n}$-connected spaces.

Finally, we shall explain the very close relationship between $L_{n}^{f}: H o_{*} \rightarrow H o_{*}$ and the $v_{n}$-periodization functor $P_{v_{n}}: H o_{*} \rightarrow H o_{*}$ of [Bou7]. For a pointed space $X$, we let $P_{(p)} X$ denote the $M$-nullification of $X$ where $M=\bigvee_{q \neq p} M(Z / q, 2)$. By [Bou7, Theorem 5.2], $P_{(p)} X$ is a partial $p$-localization of $X$ with

$$
\pi_{i} P_{(p)} X \cong \begin{cases}\pi_{i} X & \text { for } i<2 \\ \pi_{i} X / \tau_{p^{\prime}} \pi_{i} X & \text { for } i=2 \\ \pi_{i} X \otimes Z_{(p)} & \text { for } i>2\end{cases}
$$

where $\tau_{p^{\prime}} \pi_{i} X \subset \pi_{i} X$ is the subgroup of torsion prime to $p$. Since $\check{A}(n+1)=$ $A(n+1) \vee M$, there are natural maps $P_{(p)} P_{A(n+1)} \longrightarrow L_{n}^{f} X$ and $P_{A(n+1)} P_{(p)} X \longrightarrow$ $L_{n}^{f} X$, and we have

Lemma 4.10. For a space $X \in H o_{*}$ and $n \geq 0$, there are natural equivalences $P_{(p)} P_{A(n+1)} \simeq L_{n}^{f} X$ and $P_{A(n+1)} P_{(p)} X \simeq L_{n}^{f} X$. 
Proof. Since $A(n+1)$ is a simply connected space with $p$-torsion homology groups $\widetilde{H}_{*}(A(n+1) ; Z)$, the functor $P_{(p)}$ preserves the $A(n+1)$-null property, while $P_{A(n+1)}$ preserves the $M$-null property. Hence, $P_{(p)} P_{A(n+1)} X$ and $P_{A(n+1)} P_{(p)} X$ are $\check{A}(n+1)$-null, and the lemma follows easily.

4.11. Comparison of $L_{n}^{f}$ and $P_{v_{n}}$. For a space $X \in H o_{*}$, the localizations $L_{n}^{f} X$ and $P_{v_{n}} X$ are closely related by natural maps $P_{A(n+1)} X \longrightarrow L_{n}^{f} X$ and $P_{A(n+1)} X \longrightarrow P_{v_{n}} X$. The first map is obtained by Lemma 4.10 and gives $\left.\pi_{i} P_{A(n+1)}\right) X \otimes Z_{(p)} \cong \pi_{i} L_{n}^{f} X$ for $i>2$. The second map is obtained from the natural equivalences $P_{A(n+1)} X \simeq P_{\Sigma^{j} W_{n}} X$ and $P_{v_{n}} X \simeq P_{\Sigma W_{n}} X$ of [Bou7, 10.1 and 10.2], and gives $\pi_{i} P_{A(n+1)} X \cong \pi_{i} P_{v_{n}} X$ for $i>d_{n}$ by [Bou7, 7.2]. Consequently, $\pi_{i} L_{n}^{f} X \cong \pi_{i} P_{v_{n}} X \otimes Z_{(p)}$ for $i>d_{n}$. Our functors $L_{n}^{f}: H o_{*} \longrightarrow H o_{*}$ are designed to be compatible with their stable counterparts (see Proposition 5.2) and to be well-behaved above the homotopy dimension $d_{n}$ (see Lemma 12.5). We are generally not concerned with their action below the homotopy dimension $d_{n}$.

\section{The telescopic Kuhn funCtor And its ADJoint}

In this section, we state our main results on the telescopic Kuhn functor $\Phi_{n}$ : $H o_{*} \rightarrow H o^{s}$ and our new functor $\Theta_{n}: H o^{s} \rightarrow H o_{*}$, establishing strong relations between the stable and unstable telescopic homotopy theories. We begin by noting some elementary relations involving infinite loop spaces.

Proposition 5.1. For spectra $E \in H o^{s}$ and $W \in \mathcal{F}_{n}$ with $n \geq 1$, there is a natural isomorphism $v_{n}^{-1} \pi_{*}\left(\Omega^{\infty} E ; W\right) \cong v_{n}^{-1} \pi_{*}(E ; W)$.

Of course, we also have $\pi_{i}\left(\Omega^{\infty} E\right) \otimes Q \cong \pi_{i} E \otimes Q$ for $i \geq 0$. More geometrically, the infinite loop map $\Omega^{\infty} E \rightarrow \Omega^{\infty} L_{n}^{f} E$ induces a map $\lambda: L_{n}^{f} \Omega^{\infty} E \rightarrow \Omega^{\infty} L_{n}^{f} E$ since $\Omega^{\infty} L_{n}^{f} E$ is in $\mathcal{U} \mathcal{L}_{n}^{f}$. This provides natural comparison maps of telescopic towers $\lambda$ : $\left\{L_{n}^{f} \Omega^{\infty} E\right\}_{n \geq 0} \rightarrow\left\{\Omega^{\infty} L_{n}^{f} E\right\}_{n \geq 0}$ and of monocular parts $\lambda: M_{n}^{f} \Omega^{\infty} E \rightarrow \Omega^{\infty} M_{n}^{f} E$.

Proposition 5.2. For a spectrum $E$, there are equivalences

$$
\begin{aligned}
\lambda:\left(L_{n}^{f} \Omega^{\infty} E\right)\left\langle d_{n}\right\rangle \simeq\left(\Omega^{\infty} L_{n}^{f} E\right)\left\langle d_{n}\right\rangle & \text { for } n \geq 0, \\
\lambda:\left(M_{n}^{f} \Omega^{\infty} E\right)\left\langle d_{n}\right\rangle \simeq\left(\Omega^{\infty} M_{n}^{f} E\right)\left\langle d_{n}\right\rangle & \text { for } n \geq 1 .
\end{aligned}
$$

Proof. The case $n=0$ is clear. For $n \geq 1$, these maps are equivalences since they are $\bigoplus_{i=0}^{n} v_{i}^{-1} \pi_{*}$-equivalences of $\bigoplus_{i=0}^{n} v_{i}^{-1} \pi_{*}$-local spaces in $H o_{*}\left\langle d_{n}\right\rangle$ by Corollary 4.8 and Proposition 5.1.

In Kuh2 for $n \geq 1$, Kuhn introduced a remarkable functor $\phi_{n}: H o_{*} \rightarrow H o_{*}^{s}$ with $\phi_{n} \Omega^{\infty} E \simeq L_{K(n)} E$ for $E \in H o^{s}$. This greatly extended a similar result of Bou6 for $n=1$. We shall derive a telescopic Kuhn functor $\Phi_{n}: H o_{*} \rightarrow H o^{s}$ with $\Phi_{n} \Omega^{\infty} E \simeq \widehat{M}_{n}^{f} E$, and thus with $L_{K(n)} \Phi_{n} \Omega^{\infty} E \simeq L_{K(n)} E$.

Theorem 5.3 (Telescopic Kuhn theorem). For $n \geq 1$, there is a functor $\Phi_{n}$ : $H o_{*} \rightarrow H o^{s}$ such that

(i) $\Phi_{n} X$ is in $\widehat{\mathcal{M}}_{n}^{f}$ for each $X \in H o_{*}$;

(ii) for each $W \in \mathcal{F}_{n}$, there are isomorphisms

$$
v_{n}^{-1} \pi_{*}(X ; W) \cong\left[W, \Phi_{n} X\right]_{*} \cong v_{n}^{-1} \pi_{*}\left(\Phi_{n} X ; W\right)
$$

which are natural in $X \in H o_{*}$; 
(iii) for $E \in H o^{s}$, there is a natural equivalence $\Phi_{n} \Omega^{\infty} E \simeq \widehat{M}_{n}^{f} E$;

(iv) for $K, X \in H o_{*}$, there is a natural equivalence $\Phi_{n}\left(L_{n}^{f} X\right)^{K} \simeq\left(\Phi_{n} X\right)^{K}$;

(v) $\Phi_{n}$ preserves homotopy fiber squares.

A homotopy (co)fiber square in $\mathrm{Ho}_{*}$ or $\mathrm{Ho}^{s}$ is a square represented by a homotopy (co)fiber square of pointed spaces or spectra in the usual sense [BF, A.2]. A more functorial version of $5.3(\mathrm{v})$ is provided in 5.5 below. We shall prove this theorem in 13.8, and we have the following companion theorem which is the key result of this paper.

Theorem 5.4. For $n \geq 1$, there is a functor $\Theta_{n}: H o^{s} \rightarrow H o_{*}$ such that

(i) $\Theta_{n} E$ is in $\mathcal{U N}_{n}^{f}$ (and hence in $\mathcal{U} \mathcal{M}_{n}^{f}$ ) for each $E \in H o^{s}$;

(ii) for $E \in H o^{s}$ and $X \in H o_{*}$, there is a natural equivalence

$$
\operatorname{map}_{*}\left(\Theta_{n} E, L_{n}^{f} X\right) \simeq \operatorname{map}_{*}\left(E, \Phi_{n} X\right) ;
$$

(iii) for $E \in H o^{s}$, there is a natural equivalence $L_{n}^{f} \Sigma^{\infty} \Theta_{n} E \simeq M_{n}^{f} E$;

(iv) for $E \in H o^{s}$ and $K \in H o_{*}$, there is a natural equivalence $\Theta_{n}(K \wedge E) \simeq$ $L_{n}^{f}\left(K \wedge \Theta_{n} E\right)$

(v) $\Theta_{n}$ carries a homotopy cofiber square in $H o^{s}$ to an $L_{n}^{f}$-homotopy cofiber square in $\mathrm{Ho}_{*}$.

An $L_{n}^{f}$-homotopy cofiber square of pointed spaces (in $\mathcal{S S}_{*}$ ) is a commutative square

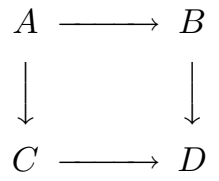

such that the map from the homotopy pushout of $C \leftarrow A \rightarrow B$ to $D$ is an $L_{n^{-}}^{f}$ equivalence; an $L_{n}^{f}$-homotopy cofiber square in $H o_{*}$ is a square represented by such a diagram of pointed spaces. We shall prove Theorem 5.4 in 13.9 .

5.5. Simplicial functoriality. As we shall see in 12.7 , the functors $\Phi_{n}: H o_{*} \rightarrow$ $H o^{s}$ and $\Theta_{n}: H o^{s} \rightarrow H o_{*}$ for $n \geq 0$ can be induced by simplicial functors $\Phi_{n}$ : $\mathcal{S S}_{*} \rightarrow \mathcal{S} p$ and $\Theta_{n}: \mathcal{S} p \rightarrow \mathcal{S S}_{*}$ on the categories $\mathcal{S S}_{*}$ and $\mathcal{S} p$ of pointed simplicial sets and spectra (see 10.3). Moreover, the functor $\Phi_{n}: \mathcal{S S}_{*} \rightarrow \mathcal{S} p$ preserves homotopy fiber squares, while $\Theta_{n}: \mathcal{S} p \rightarrow \mathcal{S S}_{*}$ carries homotopy cofiber squares to $L_{n}^{f}$-homotopy cofiber squares.

We now derive some consequences of Theorems 5.3 and 5.4.

Corollary 5.6. For $n \geq 1$, the functor $\Theta_{n}: H o^{s} \rightarrow \mathcal{U} \mathcal{L}_{n}^{f}$ is left adjoint to $\Phi_{n}$ : $\mathcal{U L}_{n}^{f} \rightarrow H o_{*}^{s}$.

Proof. This follows since there is a natural isomorphism $\left[\Theta_{n} E, L_{n}^{f} X\right] \cong\left[E, \Phi_{n} X\right]$ for $E \in H o^{s}$ and $X \in H o_{*}$ by $5.4($ ii).

Corollary 5.7. For $n \geq 1, G \in \mathcal{L}_{n}^{f}$, and $X \in H o^{s}$, there is a natural isomorphism $\widetilde{G}^{*}\left(\Theta_{n} X\right) \cong\left(\widehat{M}_{n}^{f} G\right)^{*} X$. In particular, $\left.\widehat{K(n)}\right)^{*}\left(\Theta_{n} X\right) \cong K(n)^{*} X$ and $\widehat{K(i) *}\left(\Theta_{n} X\right)=0$ for $0 \leq i<n$. Likewise, $\widetilde{K}^{*}\left(\Theta_{1} X ; \widehat{Z}_{p}\right) \cong K^{*}\left(X ; \widehat{Z}_{p}\right)$.

Proof. There are isomorphisms

$$
\left[\Theta_{n} X, \Omega^{\infty} G\right] \cong\left[X, \Phi_{n} \Omega^{\infty} G\right] \cong\left[X, \widehat{M}_{n}^{f} G\right]
$$

by Corollary 5.6 and Theorem 5.3(iii). 
Using the $K(n)_{*}$-localization functor $L_{K(n)}$, we obtain a closely related result:

Corollary 5.8. There is a natural equivalence $L_{K(n)}{ }^{\Sigma^{\infty}} \Theta_{n} X \simeq L_{K(n)} X$ for $n \geq 1$ and $X \in H o^{s}$.

Proof. This follows by applying $L_{K(n)}$ to the equivalence $L_{n}^{f} \Sigma^{\infty} \Theta_{n} X \simeq M_{n}^{f} X$ of Theorem 5.4(iii).

Thus each spectrum is $K(n)$-equivalent to a suspension spectrum. The preceding results show that $\Theta_{n}$ behaves somewhat like an infinite desuspension, and we similarly have

Corollary 5.9. For a space $A \in H o_{*}$ with a map $\nu: \Sigma^{d} A \rightarrow A$ such that $\Sigma^{\infty} \nu$ is a $v_{n}$-map in $\mathcal{F}_{n}$, there is a canonical equivalence $\Theta_{n} \Sigma^{\infty}\left(\Sigma^{2} A\right) \simeq L_{n}^{f}\left(\Sigma^{2} A\right)$.

Proof. Since $\Sigma^{\infty} \operatorname{cof} \nu$ is in $\mathcal{F}_{n+1},\left[\Sigma^{i} \operatorname{cof} \nu, X\right]$ is trivial for $X \in \mathcal{U} \mathcal{L}_{n}^{f}$ and $i \geq 1$. Hence there is an isomorphism $[\bar{A}, X] \cong v_{n}^{-1} \pi_{0}\left(X ; \Sigma^{\infty} \bar{A}\right)$ for $\bar{A}=\Sigma^{2} A$, and there are natural isomorphisms

$$
\left[L_{n}^{f} \bar{A}, X\right] \cong v_{n}^{-1} \pi_{0}\left(X ; \Sigma^{\infty} \bar{A}\right) \cong\left[\Sigma^{\infty} \bar{A}, \Phi_{n} X\right] \cong\left[\Theta_{n} \Sigma^{\infty} \bar{A}, X\right]
$$

for $X \in \mathcal{U L}_{n}^{f}$ by Theorem 5.3(ii) and Corollary 5.6. Consequently, $\Theta_{n} \Sigma^{\infty} \bar{A} \simeq$ $L_{n}^{f} \bar{A}$.

Corollary 5.10. For $n \geq 1$, we have

(i) a map $f: X \rightarrow Y$ in $H o_{*}$ is a $v_{n}^{-1} \pi_{*}$-equivalence if and only if $\Phi_{n} f: \Phi_{n} X \simeq$ $\Phi_{n} Y$

(ii) a map $g: E \rightarrow F$ in $H o^{s}$ is a $v_{n}^{-1} \pi_{*}$-equivalence if and only if $\Theta_{n} g: \Theta_{n} E \simeq$ $\Theta_{n} F$.

Proof. Part (i) follows by 5.3(i) and (ii). For part (ii), note that $\Theta_{n} g: \Theta_{n} E \simeq$ $\Theta_{n} F$ if and only if $g^{*}:\left[F, \Phi_{n} X\right] \cong\left[E, \Phi_{n} X\right]$ for each $X \in \mathcal{U} \mathcal{L}_{n}^{f}$. By 5.3(i) and (iii), this is equivalent to $g^{*}:[F, M] \cong[E, M]$ for each $M \in \widehat{\mathcal{M}}_{n}^{f}$, and hence to $\widehat{M}_{n}^{f} g: \widehat{M}_{n}^{f} E \simeq \widehat{M}_{n}^{f} F$.

Thus we see that $\Phi_{n}(Y\langle i\rangle) \simeq \Phi_{n} Y$ and $\Theta_{n}(E\langle i\rangle) \simeq \Theta_{n} E$ for each $i$. In particular, $\Phi_{n}$ kills Postnikov spaces and $\Theta_{n}$ kills Postnikov spectra. Finally, we will show that the stable monocular homotopy category $\mathcal{M}_{n}^{f}$ is a retract of the unstable monocular homotopy category $\mathcal{U N}_{n}^{f}$ in two different ways. For this we need

Lemma 5.11. For $n \geq 0$ and $X \in H o_{*}$, the map $X \rightarrow L_{n}^{f} X$ is an $S(n)_{*-}$ equivalence.

Proof. This follows as in [Bou7, 12.1] since $L_{n}^{f} X$ is the nullification of $X$ with respect to an $S(n)_{*}$-acyclic space $\check{A}(n+1)$.

Lemma 5.12. For $n \geq 1$, the functor $L_{n}^{f} \Sigma^{\infty}: \mathcal{U} \mathcal{N}_{n}^{f} \rightarrow \mathcal{M}_{n}^{f}$ is left adjoint to $\widetilde{\Omega}^{\infty}: \mathcal{M}_{n}^{f} \rightarrow \mathcal{U} \mathcal{N}_{n}^{f}$ where $\widetilde{\Omega}^{\infty} E=\left(\Omega^{\infty} E\right)\left\langle d_{n}\right\rangle$.

Proof. For a space $X \in \mathcal{U} \mathcal{N}_{n}^{f}$, the spectrum $L_{n}^{f} \Sigma^{\infty} X$ is in $\mathcal{M}_{n}^{f}$ because $X$ and $\Sigma^{\infty} X$ are $S(n-1)_{*}$-acyclic by Lemma 5.11 since $L_{n-1}^{f} X \simeq *$. For a spectrum $E \in \mathcal{M}_{n}^{f}$, the space $\widetilde{\Omega}^{\infty} E$ is in $\mathcal{U} \mathcal{N}_{n}^{f}$ since $\widetilde{\Omega}^{\infty} E \simeq\left(M_{n}^{f} \Omega^{\infty} E\right)\left\langle d_{n}\right\rangle$ by Proposition 5.2. The result now follows easily from the adjointness of $\Sigma^{\infty}$ and $\Omega^{\infty}$. 
Lemma 5.13. For $n \geq 1$, the functor $\Theta_{n}: \mathcal{M}_{n}^{f} \rightarrow \mathcal{U} \mathcal{N}_{n}^{f}$ is left adjoint to $M_{n}^{f} \Phi_{n}$ : $\mathcal{U N}_{n}^{f} \rightarrow \mathcal{M}_{n}^{f}$.

Proof. This follows by 5.4(i), 5.6, and 3.3.

By 5.3(iii), 5.4(iii), 3.3, and the above lemmas, we have

Theorem 5.14. For $n \geq 1$, the adjoint compositions

$$
\begin{aligned}
& \mathcal{M}_{n}^{f} \stackrel{\Theta_{n}}{\longleftrightarrow} \mathcal{U} \mathcal{N}_{n}^{f} \stackrel{L_{n}^{f} \Sigma^{\infty}}{\longrightarrow} \mathcal{M}_{n}^{f} \\
& \mathcal{M}_{n}^{f} \stackrel{M_{n}^{f} \Phi_{n}}{\longleftarrow} \mathcal{U} \mathcal{N}_{n}^{f} \stackrel{\tilde{\Omega}^{\infty}}{\longleftarrow} \mathcal{M}_{n}^{f}
\end{aligned}
$$

each express $\mathcal{M}_{n}^{f}$ as a categorical retract of $\mathcal{U N}_{n}^{f}$; that is, the compositions $\left(L_{n}^{f} \Sigma^{\infty}\right) \Theta_{n}$ and $\left(M_{n}^{f} \Phi_{n}\right) \widetilde{\Omega}^{\infty}$ are each equivalent to the identity functor on $\mathcal{M}_{n}^{f}$.

For $n \geq 1$, we let $\widetilde{\Omega}^{\infty} \mathcal{M}_{n}^{f} \subset \mathcal{U N}_{n}^{f}$ and $\Theta_{n} \mathcal{M}_{n}^{f} \subset \mathcal{U} \mathcal{N}_{n}^{f}$ denote the image subcategories of the faithful embeddings $\widetilde{\Omega}^{\infty}: \mathcal{M}_{n}^{f} \rightarrow \mathcal{U} \mathcal{N}_{n}^{f}$ and $\Theta_{n}: \mathcal{M}_{n}^{f} \rightarrow \mathcal{U} \mathcal{N}_{n}^{f}$. Clearly, $\widetilde{\Omega}^{\infty} \mathcal{M}_{n}^{f}$ consists of infinite loop spaces, while $\Theta_{n} \mathcal{M}_{n}^{f}$ consists of "infinite $L_{n}^{f}$-suspension spaces" since there is a natural equivalence $\Theta_{n} E \simeq L_{n}^{f} \Sigma^{k}\left(\Theta_{n} \Sigma^{-k} E\right)$ for $E \in H o^{s}$ and $k \geq 0$ by Corollary 5.4(iv). Finally, we easily see

Proposition 5.15. For spectra $E, G \in \mathcal{M}_{n}^{f}$ and $n \geq 1$, there is a natural isomorphism $\left[\Theta_{n} E, \widetilde{\Omega}^{\infty} G\right] \cong[E, G]$.

Thus a map from a space in $\Theta_{n} \mathcal{M}_{n}^{f}$ to a space in $\widetilde{\Omega}^{\infty} \mathcal{M}_{n}^{f}$ corresponds to a stable map in $\mathcal{M}_{n}^{f}$.

\section{The unstable telescope COnjeCture}

In this section, we formulate the unstable telescope conjecture and prove that it is equivalent to Ravenel's stable telescope conjecture. Our proof relies heavily on the functors $\Phi_{n}$ and $\Theta_{n}$. We start by recalling

6.1. The stable telescope conjecture for $K(n)_{*}$. For $n \geq 0$, this asserts that each $K(n)_{*}$-equivalence of spectra is a $v_{n}^{-1} \pi_{*}$-equivalence. This is equivalent to Ravenel's original telescope conjecture (see [Rav1], [MS], or 6.13 below). It holds trivially for $n=0$ and holds for $n=1$ by work of Mahowald [Mah and Miller Mil1, but has been strongly doubted for $n \geq 2$ since 1990 when Ravenel Rav2] sketched a refutation for $n=2$ and $p \geq 5$. It has the following obvious converse.

Theorem 6.2. For $n \geq 0$, each $v_{n}^{-1} \pi_{*}$-equivalence of spectra is a $K(n)_{*}$-equivalence.

Proof. This follows by 3.7 since $\langle T(n)\rangle \geq\langle T(n) \wedge K(n)\rangle=\langle K(n)\rangle$.

Thus the stable telescope conjecture would provide a perfect Whitehead theorem for $K(n)_{*}$-equivalences. To formulate an unstable telescope conjecture, we use the following concept from Bou9. For $n \geq 1$, a map $X \rightarrow Y$ in $H o_{*}$ is called a virtual $K(n)_{*}$-equivalence if it induces a $K(n)_{*}$-equivalence $(\Omega X)\langle n+2\rangle \rightarrow(\Omega Y)\langle n+2\rangle$. Results of [Bou9, Section 11] are combined to give

Theorem 6.3. For $n \geq 1$ we have

(i) each $K(n)_{*}$-equivalence of connected $H$-spaces is a virtual $K(n)_{*}$-equivalence;

(ii) an $i$-connected cover $X\langle i\rangle \rightarrow X$ of a pointed space is a virtual $K(n)_{*}$-equivalence for $i \geq 0$; 
(iii) if any two of three pointed space maps $f: X \rightarrow Y, g: Y \rightarrow Z$ and $g f: X \rightarrow Z$ are virtual $K(n)_{*}$-equivalences, then so is the third;

(iv) for a map of homotopy fiber sequences of pointed spaces

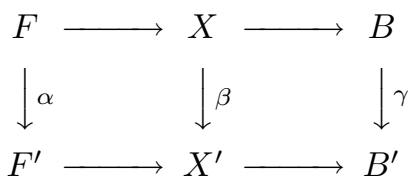

if any two of $\alpha, \beta$, and $\gamma$ are virtual $K(n)_{*}$-equivalences, then so is the third;

(v) if $f: X \rightarrow Y$ is a virtual $K(n)_{*}$-equivalence of pointed spaces, then $f\langle i\rangle$ : $X\langle i\rangle \rightarrow Y\langle i\rangle$ is a $K(n)_{*}$-equivalence for $i \geq n+2$.

Although an ordinary $K(n)_{*}$-equivalence of spaces (such as the Barratt-Priddy map $\left.K\left(\Sigma_{\infty}, 1\right) \rightarrow \Omega_{0}^{\infty} S\right)$ need not be a $v_{n}^{-1} \pi_{*}$-equivalence, we had hoped to prove

6.4. The unstable telescope conjecture for $K(n)_{*}$. For $n \geq 1$, this asserts that each virtual $K(n)_{*}$-equivalence of pointed spaces is a $v_{n}^{-1} \pi_{*}$-equivalence. This holds for $n=1$ by BBou9 11.11], but now seems likely to fail for higher $n$ by

Theorem 6.5. For each $n \geq 1$, the unstable telescope conjecture for $K(n)_{*}$ is equivalent to the stable telescope conjecture for $K(n)_{*}$.

This theorem will be proved in 6.10. The converse of the unstable telescope conjecture holds for $n=1$ by [Bou9, 11.11] but fails for $n \geq 2$; for instance, $\Omega^{\infty} K(1)$ is $v_{n}^{-1} \pi_{*}$-trivial but not virtually $K(n)_{*}$-acyclic by Theorem $6.3(\mathrm{v})$ and Bou10. However, by Bou9, 11.13], we have

Theorem 6.6. For $n \geq 1$, each $\bigoplus_{i=1}^{n} v_{i}^{-1} \pi_{*}$-equivalence of spaces is a virtual $K(n)_{*^{-}}$ equivalence.

In particular, the map $X \rightarrow L_{n}^{f} X$ is a virtual $K(n)_{*}$-equivalence for each pointed space $X$ by 4.6 . The proof of Theorem 6.5 will be based on the following lemmas.

Lemma 6.7. For $n \geq 1$, if $h: E \rightarrow E^{\prime}$ is a $K(n)_{*}$-equivalence of spectra, then $\Omega^{\infty} M_{n}^{f} h: \Omega^{\infty} M_{n}^{f} E \rightarrow \Omega^{\infty} M_{n}^{f} E^{\prime}$ is a virtual $K(n)_{*}$-equivalence of spaces.

Proof. Using the natural $\bigoplus_{i=0}^{n} v_{i}^{-1} \pi_{*}$-equivalence $\Sigma^{\infty} \Theta_{n} E \rightarrow M_{n}^{f} E$ of Theorem 5.4(iii), we obtain a commutative diagram

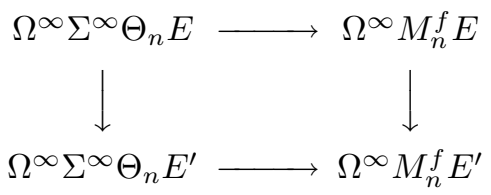

whose horizontal maps are virtual $K(n)_{*}$-equivalences by Theorem 6.6. The map $\Theta_{n} E \rightarrow \Theta_{n} E^{\prime}$ is a $K(n)_{*}$-equivalence by Corollary 5.7. Hence the left map is a $K(n)_{*}$-equivalence by [Bou4, Remark after 1.2], or [Kuh1 Proposition 2.4], and is a virtual $K(n)_{*}$-equivalence by Theorem $6.3(\mathrm{i})$. Therefore the right map is also a virtual $K(n)_{*}$-equivalence. 
The converse of the above lemma holds by

Lemma 6.8. For $n \geq 1$ and a map $h: E \rightarrow E^{\prime}$ of spectra, if $\Omega^{\infty} h: \Omega^{\infty} E \rightarrow \Omega^{\infty} E^{\prime}$ is a virtual $K(n)_{*}$-equivalence of spaces, then $h: E \rightarrow E^{\prime}$ and $\Phi_{n} \Omega^{\infty} h: \Phi_{n} \Omega^{\infty} E \rightarrow$ $\Phi_{n} \Omega^{\infty} E^{\prime}$ are $K(n)_{*}$-equivalences.

Proof. Since $\left(\Omega^{\infty} h\right)\langle n+2\rangle$ is a $K(n)_{*}$-equivalence by Theorem $6.3(\mathrm{v})$, so is the map $h\langle n+2\rangle$ by [Bou4, Proposition 1.4]. Hence the map $h$ is a $K(n)_{*}$-equivalence since any spectrum whose homotopy groups are bounded above is $K(n)_{*}$-acyclic because its rational and torsion parts are $K(n)_{*}$-acyclic by [Rav1, Theorem 4.8]. Thus, since the maps $E \rightarrow \widehat{M}_{n}^{f} E$ and $E^{\prime} \rightarrow \widehat{M}_{n}^{f} E^{\prime}$ are also $K(n)_{*}$-equivalences by Theorem 6.2 , so is $\widehat{M}_{n}^{f} h$. Hence, $\Phi_{n} \Omega^{\infty} h$ is a $K(n)_{*}$-equivalence by Theorem 5.3 (iii).

More generally, we have

Lemma 6.9. For $n \geq 1$, if $g: X \rightarrow Y$ is a virtual $K(n)_{*}$-equivalence of spaces, then $\Phi_{n} g: \Phi_{n} X \rightarrow \Phi_{n} Y$ is a $K(n)_{*}$-equivalence of spectra.

Proof. The map $L_{n}^{f} g$ is a virtual $K(n)_{*}$-equivalence by Theorem 6.6 , and hence $\operatorname{map}_{*}\left(F, L_{n}^{f} g\right)$ is a virtual $K(n)_{*}$-equivalence for each finite cell complex $F \in H o_{*}$ by Theorem 6.3. Let $A \in H o_{*}$ be a finite cell complex with a map $\nu: \Sigma^{d} A \rightarrow A$ such that $\Sigma^{\infty} A$ is a finite $p$-local spectrum of type $n$ and $\Sigma^{\infty} \nu$ is a $v_{n}$-map. Then the virtual $K(n)_{*}$-equivalence $\operatorname{map}_{*}\left(\Sigma^{2} A, L_{n}^{f} g\right)$ is an infinite loop map, where the infinite loop structure of $\operatorname{map}_{*}\left(\Sigma^{2} A, L_{n}^{f} X\right)$ comes from the natural equivalences

$$
\operatorname{map}_{*}\left(\Sigma^{2} A, L_{n}^{f} X\right) \simeq \operatorname{map}_{*}\left(\Sigma^{d+2} A, L_{n}^{f} X\right) \simeq \Omega^{d} \operatorname{map}_{*}\left(\Sigma^{2} A, L_{n}^{f} X\right)
$$

induced by $\nu: \Sigma^{d} A \longrightarrow A$. Hence $\operatorname{map}_{*}\left(\Sigma^{2} A, L_{n}^{f} g\right)$ induces a $K(n)_{*}$-equivalence $\operatorname{map}_{*}\left(\Sigma^{2} A, \Phi_{n} g\right)$ by Lemma 6.8 and Theorem 5.3(iv). Using Spanier-Whitehead duality, we conclude that $\Phi_{n} g$ is a $K(n)_{*}$-equivalence.

6.10. Proof of Theorem 6.5. Given the unstable telescope conjecture for $K(n)_{*}$, let $h: E \rightarrow E^{\prime}$ be a $K(n)_{*}$-equivalence of spectra. Then $\Omega^{\infty} \widehat{M}_{n}^{f} h$ is a virtual $K(n)_{*}$-equivalence by Lemma 6.7 , and hence is a $v_{n}^{-1} \pi_{*}$-equivalence. Thus $\widehat{M}_{n}^{f} h$ and $h$ are $v_{n}^{-1} \pi_{*}$-equivalences. Conversely, given the stable telescope conjecture for $K(n)_{*}$, let $f: X \rightarrow Y$ be a virtual $K(n)_{*}$-equivalence of spaces. Then $\Phi_{n} f$ is a $K(n)_{*}$-equivalence by Lemma 6.9 and hence is a $v_{n}^{-1} \pi_{*}$-equivalence. Thus $f$ is a $v_{n}^{-1} \pi_{*}$-equivalence by Theorem 5.3(ii).

More explicitly, a stable counterexample to the telescope conjecture will lead to a strong unstable counterexample.

Theorem 6.11. Suppose that, for some $n \geq 2$, the stable telescope conjecture fails for $K(n)_{*}$. Then, for each $j>d_{n}$, there exists a $j$-connected infinite loop space $Y$ such that $\widetilde{E(n)_{*}} Y=0, v_{n}^{-1} \pi_{*} Y \neq 0, v_{i}^{-1} \pi_{*} Y=0$ for each $i \neq n, \widetilde{S(n)_{*}} Y \neq 0$, and $\widetilde{S(n-1)}{ }_{*} Y=0$.

Proof. Choose a spectrum $E$ with $v_{n}^{-1} \pi_{*} E \neq 0$ and $K(n)_{*} E=0$, and let $Y=$ $\left(\Omega^{\infty} M_{n}^{f} E\right)\langle j\rangle$. Then clearly $v_{i}^{-1} \pi_{*} Y=0$ for $i \neq n$ and $v_{n}^{-1} \pi_{*} Y \neq 0$. Hence, $\widehat{S(n-1)_{*}} Y=0$ by Corollary 4.8 and Lemma 5.11 , and $\widetilde{S(n)_{*}} Y \neq 0$ by Bou7. 13.13]. By Lemma $6.7, \Omega^{\infty} M_{n}^{f} E$ is virtually $K(n)_{*}$-acyclic, and hence $\widetilde{K(n)_{*}} Y=0$ 
by Theorem $6.3(\mathrm{v})$ since $j>d_{n} \geq n+1$. Thus $\widetilde{E(n)_{*}} Y=0$ since $\langle E(n)\rangle=$ $\langle E(n-1) \vee K(n)\rangle \leq\langle S(n-1) \vee K(n)\rangle$.

6.12. Variants of the telescope conjecture. Since each $S(n)_{*}$-equivalence of spectra is an $E(n)_{*}$-equivalence for $n \geq 0$, there is a natural map $L_{n}^{f} X \rightarrow L_{n} X$ for $X \in H o^{s}$. Moreover, since $\langle E(n)\rangle=\langle K(0) \vee \cdots \vee K(n)\rangle$ and $\langle S(n)\rangle=$ $\langle T(0) \vee \cdots \vee T(n)\rangle$, the following conditions are equivalent for each $n \geq 0$ :

(i) the stable telescope conjecture for $K(m)_{*}$ holds for $0 \leq m \leq n$;

(ii) each $E(n)_{*}$-equivalence of spectra is an $S(n)_{*}$-equivalence;

(iii) the map $L_{n}^{f} X \rightarrow L_{n} X$ is an equivalence for each spectrum $X$.

In [Bou7, 12.3], we considered a variant of the unstable telescope conjecture asserting that, for $n \geq 0$, each $E(n)_{*}$-equivalence of spaces is an $S(n)_{*}$-equivalence. This holds for $n=1$, but will fail for some $n \geq 2$ by Theorem 6.11 if the stable telescope conjecture for $K(n)_{*}$ fails.

We conclude by showing that the stable telescope conjecture for $K(n)_{*}$ is equivalent to Ravenel's original telescope conjecture [Rav1]. For $n \geq 1$, we consider the $v_{n}$-telescope $\widehat{W}$ given by the homotopy direct limit of

$$
W \stackrel{\omega}{\longrightarrow} \Sigma^{-d} W \stackrel{\omega}{\longrightarrow} \Sigma^{-2 d} W \longrightarrow \cdots
$$

for a finite $p$-local spectrum $W$ of type $n$ with a $v_{n}$-map $\omega: \Sigma^{d} W \rightarrow W$. Ravenel's original telescope conjecture asserts that the map $\widehat{W} \rightarrow L_{n} W$ is an equivalence, and we have

Proposition 6.13. The map $\widehat{W} \rightarrow L_{n} W$ is an equivalence if and only if the stable telescope conjecture holds for $K(n)_{*}$.

Proof. Note that $\widehat{W} \simeq L_{n}^{f} W$ as in the proof of 3.6. First assume that the stable telescope conjecture holds for $K(n)_{*}$. Then the $K(n)_{*}$-equivalence $W \rightarrow L_{n} W$ is a $v_{n}^{-1} \pi_{*}$-equivalence, and is also an $S(n-1)_{*}$-equivalence since $S(n-1)_{*} W=0$ and $L_{n} W \simeq L_{n} S \wedge W$ by the smash product theorem of Hopkins and Ravenel [Rav3]. Thus $W \rightarrow L_{n} W$ is an $S(n)_{*}$-localization and $L_{n}^{f} W \simeq L_{n} W$. Conversely, assume that $L_{n}^{f} W \simeq L_{n} W$. Then

$$
\langle K(n)\rangle=\langle E(n) \wedge W\rangle=\left\langle L_{n} W\right\rangle=\left\langle L_{n}^{f} W\right\rangle=\langle S(n) \wedge W\rangle=\langle T(n)\rangle
$$

and the stable telescope conjecture holds for $K(n)_{*}$.

\section{The $E(n)_{*}$-Localizations of Postnikov $H$-Spaces}

We now determine the $E(n)_{*}$-localizations and $K(n)_{*}$-localizations of Postnikov $H$-spaces and of other $H$-spaces which are virtually $K(n)_{*}$-acyclic. We hope that our results can eventually be extended from $H$-spaces to nilpotent spaces. They depend on work of Hopkins, Ravenel, and Wilson [HRW], and will be needed in Section 8. For a nilpotent space $X$, we use the $p$-localization $X_{(p)}$, the rationalization $X_{Q}$, and the $p$-completion $\widehat{X}_{p}$ of $[\mathrm{BK}]$. We start by recalling

7.1. Modified Postnikov sections of $p$-complete nilpotent spaces. Recall that a nilpotent space is $p$-complete if and only if its homotopy groups are Ext- $p$ complete in the sense of $[\mathrm{BK}]$. For an Ext- $p$-complete nilpotent group $A$, there is a short exact sequence $0 \rightarrow \widehat{\tau} A \rightarrow A \rightarrow A / \widehat{\tau} A \rightarrow 0$ where $\widehat{\tau} A$ is the Ext-p-completion 
of the torsion subgroup of $A$, and where $A / \widehat{\tau} A$ is the greatest torsion-free Ext$p$-complete quotient of $A$. For a $p$-complete nilpotent space $Y$ and $n \geq 0$, let $Y \rightarrow \widehat{P}_{\tau}^{n+1} Y$ denote the modified $n+1$-Postnikov section with

$$
\pi_{i} \widehat{P}_{\tau}^{n+1} Y= \begin{cases}\pi_{i} Y & \text { if } i<n+1, \\ \pi_{n+1} Y / \widehat{\tau} \pi_{n+1} Y & \text { if } i=n+1, \\ 0 & \text { if } i>n+1 .\end{cases}
$$

A space will be called Postnikov when its homotopy groups vanish above some dimension. The following key result will be proved in 7.13.

Theorem 7.2. If $X$ is a connected $H$-space which is virtually $K(n)_{*}$-acyclic (e.g. Postnikov) for some $n \geq 1$, then $L_{K(n)} X \simeq \widehat{P}_{\tau}^{n+1} \widehat{X}_{p}$.

The $K(n)_{*}$-localizations of spaces are closely related to $E(n)_{*}$-localizations by

Theorem 7.3. If $X$ is a connected $H$-space or a simply connected pointed space and $n \geq 1$, then $L_{K(n)} X \simeq\left(L_{n} X\right)_{p}^{\wedge}$ and there is a homotopy fiber square

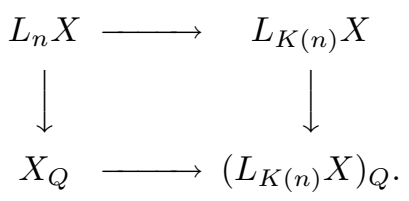

This follows by Bou10 1.6]. For a pointed nilpotent space $Y$ and $n \geq 0$, let $Y \rightarrow P_{\tau}^{n+1} Y$ denote the modified $n+1$-Postnikov section with

$$
\pi_{i} P_{\tau}^{n+1} Y= \begin{cases}\pi_{i} Y & \text { if } i<n+1, \\ \pi_{n+1} Y / \tau \pi_{n+1} Y & \text { if } i=n+1, \\ 0 & \text { if } i>n+1,\end{cases}
$$

where $\tau \pi_{n+1} Y$ is the torsion subgroup of $\pi_{n+1} Y$. We can now determine the $E(n)_{*^{-}}$ localization of a Postnikov $H$-space.

Theorem 7.4. If $X$ is a connected $H$-space which is virtually $K(n)_{*}$-acyclic (e.g. Postnikov) for some $n \geq 1$, then

$$
\pi_{i} L_{n} X= \begin{cases}\pi_{i} X \otimes Z_{(p)} & \text { if } i<n+1, \\ \left(\pi_{n+1} X / \tau \pi_{n+1} X\right) \otimes Z_{(p)} & \text { if } i=n+1, \\ \pi_{i} X \otimes Q & \text { if } i>n+1 .\end{cases}
$$

Proof. This follows since the combined square and the right square of

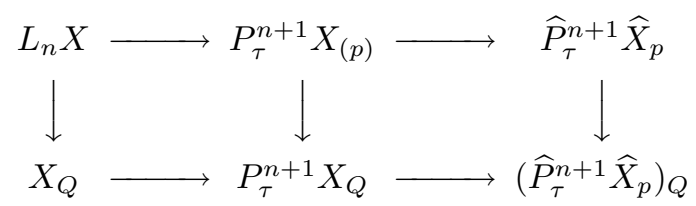

are both homotopy fiber squares by $7.2,7.3$, and the arithmetic square theorem [DDK]. Hence, the left square is also a homotopy fiber square.

To prove Theorem 7.2, we need a series of lemmas.

Lemma 7.5. If $X=K(A, m)$ for an abelian group $A$ and $m \geq 1$, then $L_{K(n)} X \simeq$ $\widehat{P}_{\tau}^{n+1} \widehat{X}_{p}$ for each $n \geq 1$. 
Proof. This follows by [Bou5, 6.3] since $K(Z / p, j)$ and $K(Z, j+1)$ are $K(n)_{*^{-}}$ nonacyclic for $1 \leq j \leq n$ and are $K(n)_{*}$-acyclic for $j>n$ by RW] and HRW] 4.4].

Lemma 7.6. For $n \geq 1$, if $X \in H o_{*}$ is a virtually $K(n)_{*}$-acyclic nilpotent space, then the map $X \rightarrow \widehat{P}_{\tau}^{n+1} \widehat{X}_{p}$ is a $K(n)_{*}$-equivalence.

Proof. The map $X \rightarrow P_{\tau}^{n+1} X$ is a $K(n)_{*}$-equivalence since the spaces $X\langle n+2\rangle$, $K\left(\pi_{n+2} X, n+2\right)$, and $K\left(\tau \pi_{n+1} X, n+1\right)$ are $K(n)_{*}$-acyclic by Theorem $6.3(\mathrm{v})$ and Lemma 7.5. The map $P_{\tau}^{n+1} X \rightarrow \widehat{P}_{\tau}^{n+1} \widehat{X}_{p}$ is a $K(n)_{*}$-equivalence since it is an $H / p_{*}$-equivalence.

Lemma 7.7. An Ext-p-complete nilpotent group $A$ is adjusted (i.e. $\widehat{\tau} A=A$ ) if and only if $\operatorname{Hom}\left(A, \widehat{Z}_{p}\right)=0$.

Proof. The upper central series quotients of $A / \widehat{\tau} A$ are torsion-free Ext-p-complete abelian groups by [BK, p. 174] and [War, p. 6]. The lemma now follows since $\widehat{Z}_{p}$ is a retract of each nontrivial torsion-free Ext-p-complete abelian group by [BK] p. 181].

Lemma 7.8. For $n \geq 1$, if $f: X \rightarrow Y$ is a $K(n)_{*}$-equivalence of pointed nilpotent spaces, then the homotopy fiber of $\widehat{f}_{p}: \widehat{X}_{p} \rightarrow \widehat{Y}_{p}$ is an $(n-1)$-connected $p$-complete space $F$ with $\pi_{n} F$ adjusted.

Proof. Since $K(Z / p, n)$ and $K\left(\widehat{Z}_{p}, n+1\right)$ are $K(n)_{*}$-local, by Lemma 7.5, we have $f^{*}: H^{i}(Y ; Z / p) \cong H^{i}(X ; Z / p)$ and $f^{*}: H^{i+1}\left(Y ; \widehat{Z}_{p}\right) \cong H^{i+1}\left(X ; \widehat{Z}_{p}\right)$ for $i \leq n$. Hence, $f_{*}: \pi_{i} \widehat{X}_{p} \rightarrow \pi_{i} \widehat{Y}_{p}$ is an isomorphism for $i<n$ and onto for $i=n$ by Bou5, 5.2]. Thus the homotopy fiber of $\widehat{f}_{p}: \widehat{X}_{p} \rightarrow \widehat{Y}_{p}$ is an $(n-1)$-connected $p$-complete space $F$ with $H^{0}\left(\widehat{Y}_{p} ; H^{n}\left(F ; \widehat{Z}_{p}\right)\right)=0$ by the Serre spectral sequence. Since $H^{n}\left(F ; \widehat{Z}_{p}\right)=\operatorname{Hom}\left(\pi_{n} F, \widehat{Z}_{p}\right)$ and since $\pi_{1} \widehat{X}_{p}$ acts nilpotently on $\pi_{n} F$ by [BK] pp. 60,61$]$, we conclude that $H^{n}\left(F ; \widehat{Z}_{p}\right)=0$. Thus the Ext-p-complete nilpotent group $\pi_{n} F$ is adjusted by Lemma 7.7, and the result follows.

For a nilpotent space $X \in H o_{*}$ and $j \geq 1$, let $X\langle j\rangle_{\tau}$ denote the homotopy fiber of the map $X \rightarrow P_{\tau}^{j} X$. Generalizing a result of Hopkins, Ravenel, and Wilson HRW], we obtain

Lemma 7.9. If $X$ is a connected Postnikov $H$-space, then the map $X\langle j\rangle_{\tau} \rightarrow X$ is a $K(n)_{*}$-monomorphism for $j, n \geq 1$.

Proof. Since $X$ is a connected $H$-space, it is a retract of $\Omega \Sigma X$, and thus is a retract of $\Omega Y$ where $Y$ is a sufficiently large Postnikov section of $\Sigma X$. Hence it suffices by naturality to show that the map $(\Omega Y)\langle j\rangle_{\tau} \rightarrow \Omega Y$ is a $K(n)_{*}$-monomorphism for each simply connected Postnikov space $Y$ and $j, n \geq 1$. We can assume that $Y$ has trivial 1-skeleton and view $Y$ as the homotopy direct limit of Postnikov sections of its finite subcomplexes. Thus by a limit argument, we can assume that $Y$ is a simply connected Postnikov space with finitely generated homotopy groups, and by [HRW] 2.3], we can also assume that $\pi_{2} Y$ is torsion. After replacing $Y$ by the homotopy fiber of $Y \rightarrow Y[1 / p]$, we obtain the desired result from Theorem 2.1 of HRW.

For a $p$-complete nilpotent space $X \in H o_{*}$ and $j \geq 1$, let $X \widehat{\langle j\rangle}_{\tau}$ denote the homotopy fiber of the map $X \rightarrow \widehat{P}_{\tau}^{j} X$. 
Lemma 7.10. If $X$ is a p-complete connected Postnikov $H$-space, then $X \widehat{\langle j\rangle}_{\tau} \rightarrow X$ is a $K(n)_{*}$-monomorphism for $j, n \geq 1$.

Proof. This follows from Lemma 7.9 since $X \widehat{\langle j\rangle_{\tau}}$ is the $p$-completion of $X\langle j\rangle_{\tau}$.

Lemma 7.11. If $u: A \rightarrow B$ is a monomorphism of p-torsion abelian groups, then $K(u, j): K(A, j) \rightarrow K(B, j)$ is a $K(n)_{*}$-monomorphism for $j, n \geq 1$.

Proof. The result holds for an inclusion $Z / p^{s} \rightarrow Z / p^{\infty}$ with $1 \leq s<\infty$ by RW] 13.1] and [HRW] 4.4], and hence holds for a finite direct sum of such inclusions by the Kunneth theorem. When $B$ is finite, we may choose such finite direct sums $\alpha: A \rightarrow I$ and $\beta: B \rightarrow J$, and then use the divisibility of $I$ and $J$ to construct a splittable monomorphism $\bar{u}: I \rightarrow J$ with $\bar{u} \alpha=\beta u$. Thus the result holds when $B$ is finite, and holds in general by a direct limit argument.

Lemma 7.12. For $n \geq 1$, if $X$ is a p-complete connected $H$-space with $X \simeq$ $\widehat{P}_{\tau}^{n+1} X$, then $X$ is $K(n)_{*}$-local.

Proof. The $K(n)_{*}$-localization $X \rightarrow L_{K(n)} X$ is a virtual $K(n)_{*}$-equivalence by Theorem 6.3(i), and thus $L_{K(n)} X \rightarrow \widehat{P}_{\tau}^{n+1} L_{K(n)} X$ is a $K(n)_{*}$-equivalence by Lemma 7.6. Hence $L_{K(n)} X$ is a retract of $\widehat{P}_{\tau}^{n+1} L_{K(n)} X$ and $L_{K(n)} X \simeq \widehat{P}_{\tau}^{n+1} L_{K(n)} X$. By Lemma 7.8, the homotopy fiber sequence $F \rightarrow X \rightarrow L_{K(n)} X$ has $\widehat{P}_{\tau}^{n} F \simeq *$ and is therefore covered by a homotopy fiber sequence $F \rightarrow X \widehat{\langle n\rangle_{\tau}} \rightarrow\left(L_{K(n)} X\right) \widehat{\langle n\rangle}_{\tau}$ which is of the form $K\left(A^{\prime}, n\right)_{p}^{\wedge} \rightarrow K(A, n)_{p}^{\wedge} \rightarrow K\left(A^{\prime \prime}, n\right)_{p}^{\wedge}$ for a short exact sequence $0 \rightarrow A^{\prime} \rightarrow A \rightarrow A^{\prime \prime} \rightarrow 0$ of $p$-torsion abelian groups obtained as follows. For any $p$-complete nilpotent space $Y$ with $\pi_{1} Y$ adjusted, there is a natural equivalence $Y \simeq\left(t_{p} Y\right)_{p}^{\wedge}$ by [Bou7, 14.1], where $t_{p} Y$ is the " $p$-torsion part" of $Y$ given by the homotopy fiber of the rationalization map $Y \rightarrow Y_{Q}$. Moreover, if $\pi_{*} Y$ is trivial except for an adjusted group $\pi_{n} Y$ and a torsion-free group $\pi_{n+1} Y$, then $t_{p} Y \simeq K\left(\pi_{n} t_{p} Y, n\right)$ where $\pi_{n} t_{p} Y$ is a $p$-torsion group. Thus the desired homotopy fiber sequence $K\left(A^{\prime}, n\right) \rightarrow K(A, n) \rightarrow K\left(A^{\prime \prime}, n\right)$ may be obtained by applying $t_{p}$ to $F \longrightarrow X \widehat{\langle n\rangle_{\tau}} \longrightarrow\left(L_{K(n)} X\right) \widehat{\langle n\rangle}_{\tau}$. Since $X \rightarrow L_{K(n)} X$ is a $K(n)_{*^{-}}$ equivalence, $K(A, n) \rightarrow K\left(A^{\prime \prime}, n\right)$ is a $K(n)_{*}$-monomorphism by Lemma 7.10 , and $K\left(A^{\prime}, n\right) \rightarrow K(A, n)$ is a $K(n)_{*}$-monomorphism by Lemma 7.11. Thus $K\left(A^{\prime}, n\right)$ is $K(n)_{*}$-acyclic and $A^{\prime}=0$ by Lemma 7.5. Hence, $F \simeq K\left(A^{\prime}, n\right)_{p}^{\wedge} \simeq *$ and $X \simeq L_{K(n)} X$.

7.13. Proof of Theorem 7.2. By Lemmas 7.6 and 7.12, the map $X \rightarrow \widehat{P}_{\tau}^{n+1} \widehat{X}_{p}$ is a $K(n)_{*}$-equivalence to a $K(n)_{*}$-local space.

\section{8. $E(n)_{*}$-LOCALIZATIONS OF INFINITE LOOP SPACES}

For a spectrum $X$, we study the $E(n)_{*}$-localization $L_{n} \Omega_{0}^{\infty} X$ of the connected infinite loop space $\Omega_{0}^{\infty} X=\left(\Omega^{\infty} X\right)\langle 0\rangle$. There is a natural comparison map $L_{n} \Omega_{0}^{\infty} X \rightarrow$ $\Omega_{0}^{\infty} L_{n} X$ since the space $\Omega_{0}^{\infty} L_{n} X$ is $E(n)_{*}$-local. In [Bou4], we showed that $L_{1} \Omega_{0}^{\infty} X$ is given by $\Omega_{0}^{\infty} L_{1} X$ with low dimensional modifications. We now obtain a similar result for $L_{2} \Omega_{0}^{\infty} X$, and more generally for $L_{n} \Omega_{0}^{\infty} X$ when $L_{n-1}^{f} X \simeq L_{n-1} X$. The latter condition would be automatic if the telescope conjecture were valid, and holds when $X$ is a $B P$-module spectrum or any other $B P$-cotrivial spectrum (see 6.12 and 8.9 ). 
Theorem 8.1. For $n \geq 1$ and a spectrum $X$ with $L_{n-1}^{f} X \simeq L_{n-1} X$, the natural map

$$
L_{n} \Omega_{0}^{\infty} X \rightarrow \Omega_{0}^{\infty} L_{n} X \times{ }_{P_{\tau}^{n+1} \Omega_{0}^{\infty} L_{n} X} P_{\tau}^{n+1} \Omega_{0}^{\infty} X_{(p)}
$$

is a $\pi_{i}$-isomorphism for $i \neq n+1$ and is a $\pi_{n+1}$-monomorphism. The condition $L_{n-1}^{f} X \simeq L_{n-1} X$ holds for all spectra when $n \leq 2$, and the natural map is always an equivalence when $n=1$.

This will be proved in 8.6.

Corollary 8.2. For $n \geq 1$ and a spectrum $X$ with $L_{n-1}^{f} X \simeq L_{n-1} X$, the natural map

$$
L_{K(n)} \Omega_{0}^{\infty} X \rightarrow\left(\Omega_{0}^{\infty} L_{n} X\right)_{p}^{\wedge} \times_{\widehat{P}_{\tau}^{n+1}\left(\Omega_{0}^{\infty} L_{n} X\right)_{p}^{\wedge}} \widehat{P}_{\tau}^{n+1}\left(\Omega_{0}^{\infty} X\right)_{p}^{\wedge}
$$

is a $\pi_{i}$-isomorphism for $i \neq n+1, n+2$ and is a $\pi_{n+2}$-monomorphism. The natural map is always an equivalence when $n=1$.

This follows from Theorems 8.1 and 7.3. To prove Theorem 8.1, we need three lemmas.

Lemma 8.3. For $n \geq 1$, if $h: X \rightarrow Y$ is a $K(n)_{*}$-equivalence and an $L_{n-1}^{f}$ equivalence of spectra, then $\Omega^{\infty} h: \Omega^{\infty} X \rightarrow \Omega^{\infty} Y$ is a virtual $K(n)_{*}$-equivalence of spaces.

Proof. In the map of fiber sequences

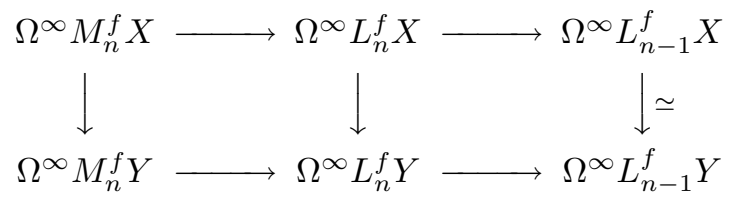

the vertical arrows are virtual $K(n)_{*}$-equivalences by Lemma 6.7 and Theorem $6.3\left(\right.$ iv). Since the maps $\Omega^{\infty} X \longrightarrow \Omega^{\infty} L_{n}^{f} X$ and $\Omega^{\infty} Y \longrightarrow \Omega^{\infty} L_{n}^{f} Y$ are also virtual $K(n)_{*}$-equivalences by Theorem 6.6 , so is the map $\Omega^{\infty} X \longrightarrow \Omega^{\infty} Y$.

Lemma 8.4. For $n \geq 1$, if $X$ is a spectrum with $L_{n-1}^{f} X \simeq L_{n-1} X$, then the map $L_{n} \Omega_{0}^{\infty} X \rightarrow \Omega_{0}^{\infty} L_{n} X$ is a $\pi_{i}$-isomorphism for $i>n+1$ and a $\pi_{n+1}$-monomorphism.

Proof. Since $S(n-1)$ and $E(n)$ are smashing with $\langle E(n-1)\rangle=\langle S(n-1) \wedge E(n)\rangle$, we have $L_{n-1} X \simeq L_{n-1}^{f} L_{n} X$ and hence $L_{n-1}^{f} X \simeq L_{n-1}^{f} L_{n} X$. Thus Lemma 8.3 shows that the map of spectra $X \rightarrow L_{n} X$ induces a virtual $K(n)_{*}$-equivalence of spaces $\Omega_{0}^{\infty} X \rightarrow \Omega_{0}^{\infty} L_{n} X$. Since the $E(n)_{*}$-localization $\Omega_{0}^{\infty} X \rightarrow L_{n} \Omega_{0}^{\infty} X$ is also a virtual $K(n)_{*}$-equivalence by Theorem $6.3(\mathrm{i})$, so is the map $L_{n} \Omega_{0}^{\infty} X \rightarrow \Omega_{0}^{\infty} L_{n} X$. Thus its homotopy fiber $F$ is virtually $K(n)_{*}$-acyclic. Since $F$ is an $E(n)_{*}$-local, $p$-torsion, infinite loop space by [Bou4], we have $F \simeq P_{\tau}^{n+1} F$ by Theorem 7.4, and $\pi_{i} F=0$ for $i \geq n+1$.

Lemma 8.5. For $n \geq 1$, each $E(n)_{*}$-equivalence of $p$-local connected $H$-spaces is a $P_{\tau}^{n+1}$-equivalence.

Proof. Let $\mathcal{C} \subset \mathrm{Ho}_{*}$ be the full subcategory of all $p$-local connected $H$-spaces $Y$ with $P_{\tau}^{n+1} Y \simeq Y$. Since each $Y \in \mathcal{C}$ is $E(n)_{*}$-local by Theorem 7.4 , an $E(n)_{*^{-}}$ equivalence $f: A \rightarrow B$ in $H o_{*}$ induces a bijection $f^{*}:[B, Y] \cong[A, Y]$, and thus induces a bijection $\left(P_{\tau}^{n+1} f\right)^{*}:\left[P_{\tau}^{n+1} B, Y\right] \cong\left[P_{\tau}^{n+1} A, Y\right]$ for all $Y \in \mathcal{C}$. When 
$A$ and $B$ are $p$-local connected $H$-spaces, so are $P_{\tau}^{n+1} A$ and $P_{\tau}^{n+1} B$, and thus $P_{\tau}^{n+1} f: P_{\tau}^{n+1} A \rightarrow P_{\tau}^{n+1} B$ is an equivalence in $\mathcal{C}$ by the $Y$ oneda lemma.

8.6. Proof of Theorem 8.1. The theorem follows for $n=1$ by Bou4. Since $L_{1}^{f} X \simeq L_{1} X$ holds for all $X$ (see 6.12), we may assume $n \geq 2$ with $L_{n-1}^{f} X \simeq$ $L_{n-1} X$. Thus the map $L_{n} \Omega_{0}^{\infty} X \rightarrow \Omega_{0}^{\infty} L_{n} X$ is a $\pi_{i}$-isomorphism for $i>n+1$ and a $\pi_{n+1}$-monomorphism by Lemma 8.4, and the map $\Omega_{0}^{\infty} X_{(p)} \rightarrow L_{n} \Omega_{0}^{\infty} X$ is a $P_{\tau}^{n+1}$-equivalence by Lemma 8.5. The theorem now follows easily.

8.7. A counterexample. Unfortunately, when $n=2$, the natural map of Theorem 8.1 may actually fail to be a $\pi_{3}$-isomorphism. To see this, note that for $1 \leq j \leq \infty$, the map $\Sigma^{\infty} M\left(Z / p^{j}, 3\right)\langle 3\rangle \rightarrow \Sigma^{\infty} M\left(Z / p^{j}, 3\right)$ is carried to an equivalence by $\Omega_{0}^{\infty} L_{2}, P_{\tau}^{3} \Omega_{0}^{\infty} L_{2}$, and $P_{\tau}^{3} \Omega_{0}^{\infty}$, but not by $L_{2} \Omega_{0}^{\infty}$, because we have

Lemma 8.8. For $1 \leq j \leq \infty$, the map $Q M\left(Z / p^{j}, 3\right)\langle 3\rangle \rightarrow Q M\left(Z / p^{j}, 3\right)$ of spaces is not a $K(2)_{*}$-monomorphism.

Proof. In the principal fiber sequence

$$
Q M\left(Z_{p^{\infty}}, 2\right) \longrightarrow K\left(Z_{p^{\infty}}, 2\right) \longrightarrow Q M\left(Z_{p^{\infty}}, 3\right)\langle 3\rangle,
$$

$K(2)_{*} Q M\left(Z_{p^{\infty}}, 2\right) \cong K(2)_{*} Q S^{3}$ is an exterior algebra on odd dimensional generators by Kashiwabara [Kas. Thus since $K(2)_{*} K\left(Z_{p^{\infty}}, 2\right)$ is concentrated in even dimensions, a bar spectral sequence argument shows that the map $K\left(Z_{p^{\infty}}, 2\right) \longrightarrow$ $Q M\left(Z_{p^{\infty}}, 3\right)\langle 3\rangle$ is a $K(2)_{*}$-monomorphism. Since the map $K\left(Z_{p^{j}}, 2\right) \rightarrow K\left(Z_{p^{\infty}}, 2\right)$ is also a $K(2)_{*}$-monomorphism by Lemma 7.11 , we see that the map $K\left(Z / p^{j}, 2\right) \longrightarrow$ $Q M\left(Z / p^{j}, 3\right)\langle 3\rangle$ is a nontrivial $K(2)_{*}$-monomorphism for $1 \leq j \leq \infty$. Hence, the map $Q M\left(Z / p^{j}, 3\right)\langle 3\rangle \longrightarrow Q M\left(Z / p^{j}, 3\right)$ cannot be a $K(2)_{*}$-monomorphism.

Finally, we show that our results on $L_{n} \Omega^{\infty} X$ and $L_{K(n)} \Omega^{\infty} X$ apply whenever $X$ is a $B P$-cotrivial spectrum in the sense of 2.8. We note that the $B P$-cotrivial spectra include the $B P$-module spectra and the $B P$-nilpotent spectra in the sense of [Bou2, 3.7].

Lemma 8.9. If $X$ is a BP-cotrivial spectrum and $n \geq 0$, then $L_{n}^{f} X \simeq L_{n} X$.

Proof. Let $\mathcal{C}$ be the class of all spectra $X$ with $L_{n}^{f} X \simeq L_{n} X$. Since $L_{n}^{f} X \simeq X \wedge L_{n}^{f} S$, $L_{n} X \simeq X \wedge L_{n} S$, and $B P \wedge L_{n}^{f} S \simeq B P \wedge L_{n} S$ by [Rav4, 2.7], we have (i) $\mathcal{C}$ contains $B P$; (ii) $\mathcal{C}$ is closed under arbitrary wedges; and (iii) $\mathcal{C}$ contains all three spectra in a cofiber sequence when it contains any two. Hence, $\mathcal{C}$ contains the $B P$-cotrivial spectra by 2.8 .

\section{Model CATEgories AND NULLifications}

To prepare for the proofs of Theorems 5.3 and 5.4, we now give a much improved version of the Bousfield-Friedlander $\overline{\mathrm{BF}}$ construction of new model categories from old ones with " $Q$-structures," and then show that each nullification functor determines a proper model category structure on spaces. By a model category, we mean a closed model category in Quillen's original sense Qui. We refer the reader to [DS], [GJ], [Hir], and [Hov] for good recent or forthcoming treatments of model categories, and we note that our results are fully compatible with the slightly refined definitions of a "model category" used in $\mathrm{Hir}$, $\mathrm{Hov}$, and elsewhere. We shall need 
9.1. Proper model categories. A model category $\mathcal{C}$ is called:

(i) right proper if every pullback of a weak equivalence along a fibration is a weak equivalence;

(ii) left proper if every pushout of a weak equivalence along a cofibration is a weak equivalence;

(iii) proper if it is both right proper and left proper.

As explained in $\mathrm{BF}$ or Hir, an elementary theory of homotopy pullbacks (resp. pushouts) is available in a right (resp. left) proper model category. All model categories used in this paper will turn out to be proper.

9.2. $Q$-structures on proper model categories. Let $\mathcal{C}$ be a proper model category with a functor $Q: \mathcal{C} \rightarrow \mathcal{C}$ and natural transformation $\alpha: 1 \rightarrow Q$ satisfying the following axioms:

(A1) if $f: X \rightarrow Y$ is a weak equivalence in $\mathcal{C}$, then so is $Q f: Q X \rightarrow Q Y$;

(A2) for each $X \in \mathcal{C}$, the maps $\alpha, Q \alpha: Q X \rightarrow Q Q X$ are weak equivalences;

(A3) for a pullback square

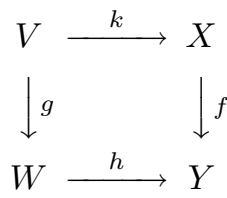

in $\mathcal{C}$, if $f$ is a fibration of fibrant objects such that $\alpha: X \rightarrow Q X, \alpha: Y \rightarrow Q Y$, and $Q h: Q W \rightarrow Q Y$ are weak equivalences, then $Q k: Q V \rightarrow Q X$ is a weak equivalence.

A map $f: X \rightarrow Y$ in $\mathcal{C}$ will be called a $Q$-equivalence if $Q f: Q X \rightarrow Q Y$ is a weak equivalence, a $Q$-cofibration if $f$ is a cofibration, and a $Q$-fibration if $f$ has the right lifting property for $Q$-trivial cofibrations (the $Q$-cofibrations which are $Q$-equivalences). We let $\mathcal{C}^{Q}$ denote the category $\mathcal{C}$ equipped within its $Q$ equivalences, $Q$-cofibrations, and $Q$-fibrations. The following theorem is a much improved version of Theorem A.7 from $\mathrm{BF}$. Our present axiom (A3) replaces the hard-to-verify axiom (A.6), which involved general $Q$-fibrations and included a dual pushout condition.

Theorem 9.3. If $\mathcal{C}$ is a proper model category with a functor $Q: \mathcal{C} \rightarrow \mathcal{C}$ and transformation $\alpha: 1 \rightarrow Q$ satisfying (A1), (A2), and (A3), then $\mathcal{C}^{Q}$ is also a proper model category. Moreover, a map $f: X \rightarrow Y$ in $\mathcal{C}$ is a $Q$-fibration if and only if $f$ is a fibration and

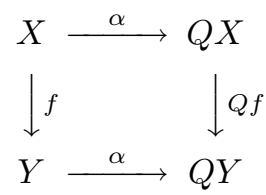

is a homotopy fiber square in $\mathcal{C}$.

Proof. The proof proceeds as in $[\mathrm{BF}$, Appendix A] with a new construction of factorizations into $Q$-trivial cofibrations and $Q$-fibrations, and with a new verification 
of properness. To factor a map $f: X \rightarrow Y$, take the commutative diagram

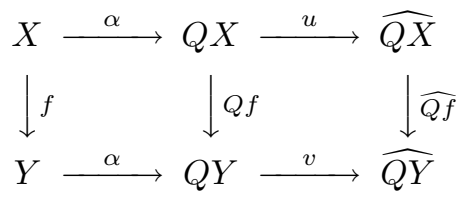

where $u$ and $v$ are trivial cofibrations and $\widehat{Q f}: \widehat{Q X} \rightarrow \widehat{Q Y}$ is a fibration of fibrant objects. Then $\widehat{Q f}$ is a $Q$-fibration by [BF, (A.8)], and $\widehat{Q f}$ pulls back along the $Q$-equivalence $Y \rightarrow \widehat{Q Y}$ to give a $Q$-fibration $g: E \rightarrow Y$ such that the map $E \rightarrow \widehat{Q X}$ is a $Q$-equivalence by (A3). Hence the map $X \rightarrow E$ is a $Q$-equivalence and has a factorization into a $Q$-trivial cofibration $j: X \rightarrow D$ and a trivial fibration $k: D \rightarrow E$. Now $f=(g k) j$ is the desired factorization of $f$. The resulting model category $\mathcal{C}^{Q}$ is left proper since the pushout of a $Q$-equivalence along a cofibration may be obtained by successively pushing out a $Q$-trivial cofibration and a weak equivalence. It is right proper by (A3) and the following lemma.

Lemma 9.4. A model category $\mathcal{D}$ is right proper if every pullback of a weak equivalence along a fibration of fibrant objects in $\mathcal{D}$ is a weak equivalence.

Proof. Let

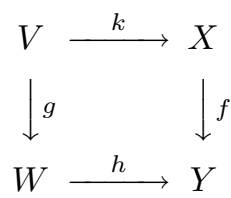

be a pullback of a weak equivalence $h$ along a fibration $f$ in $\mathcal{D}$. Construct a fibration $\widehat{f}: \widehat{X} \rightarrow \widehat{Y}$ of fibrant objects $\widehat{X}$ and $\widehat{Y}$ with trivial cofibrations $u: X \rightarrow \widehat{X}$ and $v: Y \rightarrow \widehat{Y}$ such that $v f=\widehat{f} u$. Let $f^{\prime}: X^{\prime} \rightarrow Y$ and $g^{\prime}: V^{\prime} \rightarrow W$ be the induced fibrations of $\widehat{f}$. Since the maps $X^{\prime} \rightarrow \widehat{X}$ and $V^{\prime} \rightarrow \widehat{X}$ are weak equivalences by hypothesis, so are the maps $V^{\prime} \rightarrow X^{\prime}$ and $X \rightarrow X^{\prime}$. By Ken Brown's lemma (see DS 9.9]), the functor $h^{*}: \mathcal{D} \downarrow Y \rightarrow \mathcal{D} \downarrow W$ preserves weak equivalences of fibrant objects. Thus $h^{*}$ carries the weak equivalence $f \rightarrow f^{\prime}$ to a weak equivalence $g \rightarrow g^{\prime}$. Hence the maps $V \rightarrow V^{\prime}$ and $V \rightarrow X$ are weak equivalences.

Remark 9.5. Theorem 9.3 remains valid when "proper" is replaced by "right proper." However, the proof then requires another new argument to verify Lemma A.8(iii) of $[\mathrm{BF}$. This can be given using a lemma of Dan Kan proved in Hir 11.1.16].

9.6. Simplicial model categories. By a simplicial model category, we mean a closed simplicial model category in Quillen's original sense Qui, II §2], but without any finiteness conditions on simplicial sets in the axiom SM0. In particular, a simplicial model category $\mathcal{C}$ is enriched over the category $\mathcal{S S}$ of simplicial sets and is both tensored and cotensored (see e.g. Bor $)$. For $X, Y \in \mathcal{C}$ and $K \in \mathcal{S S}$, we write $\operatorname{map}(X, Y) \in \mathcal{S S}, X \otimes K \in \mathcal{C}$, and $X^{K}=\operatorname{map}(K, X) \in \mathcal{C}$ for the mapping space, tensor, and cotensor. Theorem 9.3 now extends to

Theorem 9.7. Under the hypotheses of Theorem 9.3, if $\mathcal{C}$ is a proper simplicial model category, then so is $\mathcal{C}^{Q}$. 
Proof. It suffices to verify Quillen's axiom SM7(b) for $\mathcal{C}^{Q}$. The only nontrivial step is to show that if $A \rightarrow B$ is a $Q$-trivial cofibration, then so is

$$
F_{n}: B \otimes \partial \Delta^{n} \coprod_{A \otimes \partial \Delta^{n}} A \otimes \Delta^{n} \longrightarrow B \otimes \Delta^{n}
$$

for $n \geq 0$. Let $\Lambda_{0}^{n} \subset \Delta^{n}$ and $\partial \Delta^{n} \subset \Delta^{n}$ be the 0-horn and boundary of the standard $n$-simplex. In the pushout square

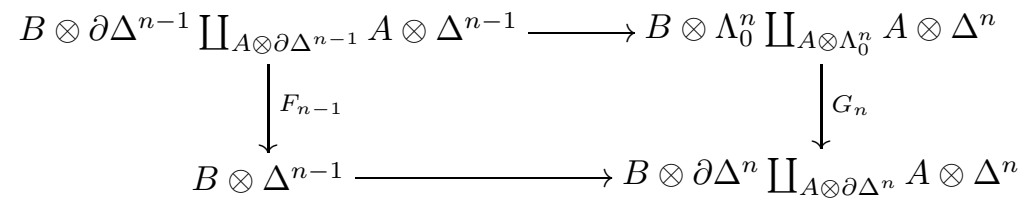

we can assume inductively that $F_{n-1}$ is a $Q$-trivial cofibration and deduce that $G_{n}$ is also a $Q$-trivial cofibration. Since $\mathcal{C}$ is a simplicial model category and $\Lambda_{0}^{n} \rightarrow \Delta^{n}$ is a trivial cofibration, we see that $F_{n} G_{n}$ is a trivial cofibration. Likewise, $F_{n}$ is a cofibration. Hence $F_{n}$ is a $Q$-trivial cofibration.

9.8. The nullification functors. We now work in the proper simplicial model category $\mathcal{S} \mathcal{S}_{*}$ of pointed spaces (i.e. pointed simplicial sets). For each pointed space $W$, we consider the $W$-nullification (=W-periodization) functor $P_{W}: \mathcal{S S}_{*} \rightarrow \mathcal{S S}_{*}$ and transformation $\alpha: 1 \rightarrow P_{W}$ of [Bou7, 2,10] or [Dro, which induce the $W$ nullification functor $P_{W}: H o_{*} \rightarrow H o_{*}$ and transformation $\alpha: 1 \rightarrow P_{W}$ of 4.1. Using the terminology of 9.2 , we have

Theorem 9.9. If $W$ is a pointed space, then $\mathcal{S S}_{*}^{P_{W}}$ is a proper simplicial model category. Moreover, a map $f: X \rightarrow Y$ in $\mathcal{S S}_{*}$ is a $P_{W}$-fibration if and only if $f$ is a fibration and

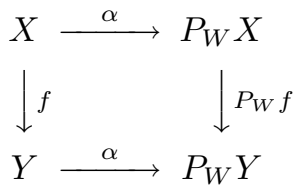

is a homotopy fiber square in $\mathcal{S S}_{*}$.

Proof. It suffices by Theorem 9.7 to verify our three axioms for $P_{W}: \mathcal{S S}_{*} \rightarrow \mathcal{S S}_{*}$. Axioms (A1) and (A2) follow since $P_{W}$ induces an idempotent functor on $\mathrm{Ho}_{*}$. For (A3), we must show that if

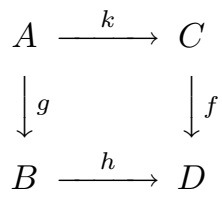

is a pullback square in $\mathcal{S S}_{*}$ where $f$ is a fibration of $W$-null fibrant spaces and where $h$ is a $P_{W}$-equivalence, then $k$ is a $P_{W}$-equivalence. When $C$ and $D$ are connected, this follows by [Bou7, 4.8(i)] since the homotopy fiber of $h$ is $P_{W}$-acyclic by [Bou7, 4.3]. The result now follows in general since the functor $P_{W}: \mathcal{S S}_{*} \rightarrow \mathcal{S S}_{*}$ comes from a functor $P_{W}: \mathcal{S S} \rightarrow \mathcal{S S}$ which preserves disjoint unions when $W$ is connected, and which takes contractible values when $W$ is not connected. 
9.10. The homotopy category $\mathcal{P}_{W}$. For a pointed space $W$, we let $\mathcal{P}_{W} \subset H o_{*}$ be the full subcategory of $W$-null spaces (see 4.1). This is the homotopy category of fibrant cofibrant objects in $\mathcal{S S}_{*}^{P_{W}}$, and is thus canonically equivalent to the Quillen homotopy category $\mathrm{Ho}\left(\mathcal{S S}_{*}^{P_{W}}\right)$ obtained by inverting the $P_{W}$-equivalences in $\mathcal{S S}_{*}$.

Remark 9.11. The $W$-nullification functors $P_{W}$ are special cases of the $f$-localization functors $L_{f}$ constructed for arbitrary maps $f$ of pointed spaces in [Bou7], [Bou9, [Dro, and [Hir]. In general, there is a left proper simplicial model category structure on $\mathcal{S S}_{*}$ whose weak equivalences are the $L_{f}$-equivalences and whose cofibrations are the usual injections (see [Bou9, 4.6] or [Hir]). However, this model category will not be right proper unless $L_{f}$ is equivalent to a nullification, as we now explain. By [Bou9 Theorem 4.4], for an arbitrary map $f$ of pointed spaces, there exists a pointed space $A(f)$ such that $P_{A(f)}$ and $L_{f}$ have the same acyclic spaces. Moreover, each $P_{A(f)}$-equivalence of pointed spaces is an $L_{f}$-equivalence. Now suppose that the model category structure for $L_{f}$ on $\mathcal{S S}_{*}$ is right proper. Then for a pointed connected space $X$, the homotopy fiber of $X \rightarrow L_{f} X$ is $L_{f}$ acyclic, since a path fibration over $L_{f} X$ is an $L_{f}$-fibration by [BF, A.3(iii)]. Hence, this homotopy fiber is also $P_{A(f)}$-acyclic, and $X \rightarrow L_{f} X$ is a $P_{A(f)}$-equivalence by [Bou7, Corollary 4.8(i)]. Thus each $L_{f}$-equivalence of pointed connected spaces is a $P_{A(f)}$-equivalence, and this generalizes to nonconnected spaces as in the proof of Theorem 9.9. Hence $L_{f}$ is equivalent to $P_{A(f)}$.

\section{The FunCtors $\Phi_{A}$ AND $\Theta_{A}$}

To prepare for the proofs of Theorems 5.3 and 5.4, we now introduce a Kuhn functor $\Phi_{A}: \mathcal{P}_{W} \rightarrow H o^{s}$ and its left adjoint $\Theta_{A}: H o^{s} \rightarrow \mathcal{P}_{W}$ for a " $P_{W}$-cospectrum" $A$, where $W$ is a fixed pointed space. These functors will be constructed more generally in Section 11. To establish notation and terminology, we first discuss

10.1. Pointed simplicial Quillen functors. A pointed simplicial model category $\mathcal{C}$ is automatically enriched, tensored, and cotensored over $\mathcal{S S}_{*}$. For $X, Y \in \mathcal{C}$ and $K \in \mathcal{S S}_{*}$, we let $\operatorname{map}_{*}(X, Y) \in \mathcal{S S}_{*}, X \wedge K \in \mathcal{C}$, and $X^{K}=\operatorname{map}_{*}(K, X) \in \mathcal{C}$ denote the pointed mapping space, tensor, and cotensor in $\mathcal{C}$. The Quillen homotopy category $\mathrm{HoC}$ is consequently enriched, tensored, and cotensored over $H o\left(\mathcal{S S}_{*}\right)=H o_{*}$. For pointed simplicial model categories $\mathcal{C}$ and $\mathcal{D}$, a pair of functors $F: \mathcal{C} \rightarrow \mathcal{D}$ and $G: \mathcal{D} \rightarrow \mathcal{C}$ are called pointed simplicial left and right Quillen functors if $F$ is pointed simplicially left adjoint to $G$ and the following equivalent conditions are satisfied:

(i) $F$ preserves cofibrations and $G$ preserves fibrations;

(ii) $F$ preserves cofibrations and trivial cofibrations;

(iii) $G$ preserves fibrations and trivial fibrations.

Thus, in the language of enriched category theory (see e.g. [Bor, p. 340]), $F$ and $G$ are $\mathcal{S S}_{*}$-functors such that $F$ is left $\mathcal{S S}_{*}$-adjoint to $G$ and they satisfy conditions (i)-(iii). By adjunction arguments, there are natural isomorphisms $F(X \wedge K) \cong$ $F(X) \wedge K$ and $G\left(Y^{K}\right) \cong(G Y)^{K}$ for $X \in \mathcal{C}, Y \in \mathcal{D}$, and $K \in \mathcal{S S}_{*}$. Moreover, $F$ (resp. $G$ ) preserves weak equivalences of cofibrant (resp. fibrant) objects by Ken Brown's lemma DS 9.9]. Thus, $F$ preserves homotopy cofiber squares of cofibrant objects, while $G$ preserves homotopy fiber squares of fibrant objects.

By Qui, I.4], $F$ has a total left derived functor $\mathbf{L} F: H o \mathcal{C} \rightarrow H o \mathcal{D}$, and $G$ has a total right derived functor $\mathbf{R} G: H o \mathcal{D} \rightarrow H o \mathcal{C}$, where $\mathbf{L} F$ is left adjoint to 
RG. Moreover, there are natural equivalences $\mathbf{L} F(X \wedge K) \simeq \mathbf{L} F(X) \wedge K$ and $\mathbf{R} G\left(Y^{K}\right) \simeq(\mathbf{R} G Y)^{K}$ for $X \in H o \mathcal{C}, Y \in H o \mathcal{D}$, and $K \in H o_{*}$. The functor $\mathbf{L} F$ preserves homotopy cofiber squares, while $\mathbf{R} G$ preserves homotopy fiber square.

The pointed simplicial left and right Quillen functors $F: \mathcal{C} \rightarrow \mathcal{D}$ and $G: \mathcal{D} \rightarrow \mathcal{C}$ are called Quillen equivalences when, for each cofibrant $X \in \mathcal{C}$ and fibrant $Y \in \mathcal{D}$, a map $F X \rightarrow Y$ is a weak equivalence if and only if its adjoint $X \rightarrow G Y$ is a weak equivalence. This implies that the functors $\mathbf{L} F: H o \mathcal{C} \leftrightarrows H o \mathcal{D}: \mathbf{R} G$ are adjoint equivalences. To construct our Quillen functors, we use

10.2. $P_{W}$-cospectra. A cospectrum $A$ consists of spaces $A^{n} \in \mathcal{S S}_{*}$ for $n \geq 0$ and structure maps $\sigma: A^{n+1} \wedge S^{1} \rightarrow A^{n}$ where $S^{1}=\Delta^{1} / \partial \Delta^{1}$. We call $A$ a $P_{W^{-}}$ cospectrum if $\sigma: A^{n+1} \wedge S^{1} \rightarrow A^{n}$ is a $P_{W}$-equivalence for $n \geq 0$. This implies that $A^{0}$ is an "infinite suspension space" in $P_{W}$-homotopy theory. We remark that a $P_{W}$-equivalence $J \wedge S^{d} \rightarrow J$, for a space $J$ and $d>0$, can be used to construct a $P_{W}$-cospectrum with successive spaces $J, J \wedge S^{d-1}, \ldots, J \wedge S^{1}, J, \ldots$ where $S^{d}=S^{1} \wedge \ldots \wedge S^{1}$.

10.3. Spectra. A spectrum $E$ consists of spaces $E_{n} \in \mathcal{S S}_{*}$ for $n \geq 0$ with structure maps $\sigma: S^{1} \wedge E_{n} \rightarrow E_{n+1}$. It has homotopy groups $\pi_{*} E=\operatorname{colim}_{n} \pi_{*+n} E_{n}$. By $[\mathrm{BF}]$ or [HSS], there is a proper pointed simplicial model category ${ }^{n} \mathcal{S} p$ of spectra, where a map $f: E \rightarrow F$ is a weak equivalence when $f_{*}: \pi_{*} E \cong \pi_{*} F$, a cofibration when $f$ induces cofibrations $E_{0} \rightarrow F_{0}$ and $E_{n+1} \coprod_{S^{1} \wedge E_{n}} S^{1} \wedge F_{n} \rightarrow F_{n+1}$ for $n \geq 0$, and a fibration when $f$ induces fibrations $E_{n} \rightarrow F_{n}$ and homotopy fiber squares

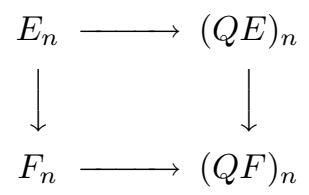

in $\mathcal{S S}_{*}$ for $n \geq 0$ using an omegafication functor $Q: \mathcal{S} p \longrightarrow \mathcal{S} p$. A map $f: E \rightarrow F$ is a trivial fibration in $\mathcal{S} p$ if and only if it induces trivial fibrations $E_{n} \rightarrow F_{n}$ in $\mathcal{S S}_{*}$ for $n \geq 0$. We may identify $H o(\mathcal{S} p)$ with the stable homotopy category $H o^{s}$.

10.4. The functor $\phi_{A}: \mathcal{S S}_{*}^{P_{W}} \rightarrow \mathcal{S} p$. For a $P_{W}$-cospectrum $A$, there is a pointed simplicial functor $\phi_{A}: \mathcal{S}_{*} P_{W} \rightarrow \mathcal{S} p$ where $\phi_{A}(X)$ is the spectrum with $\phi_{A}(X)_{n}=$ $X^{A^{n}}$ for $n \geq 0$ and with the induced structure maps. The functor $\phi_{A}$ has a left adjoint pointed simplicial functor $\theta_{A}: \mathcal{S} p \rightarrow \mathcal{S S}_{*}^{P_{W}}$ carrying a spectrum $E$ to the pointed space $\theta_{A}(E)$, which may be constructed as the quotient of $\bigvee_{n \geq 0}\left(A^{n} \wedge E_{n}\right)$ by the relations given by

$$
A^{n+1} \wedge E_{n+1} \stackrel{1 \wedge \sigma}{\longleftarrow} A^{n+1} \wedge S^{1} \wedge E_{n} \stackrel{\sigma \wedge 1}{\longrightarrow} A^{n} \wedge E_{n}
$$

for $n \geq 0$. To show that $\theta_{A}$ and $\phi_{A}$ are left and right Quillen functors, we need

Lemma 10.5. Let $T: \mathcal{C} \rightarrow \mathcal{D}$ be a functor of model categories. If $\mathcal{C}$ is right proper and $T$ preserves pullbacks, trivial fibrations, and fibrations of fibrant objects, then $T$ preserves arbitrary fibrations. 
Proof. For a fibration $f: X \rightarrow Y$ in $\mathcal{C}$, construct a square

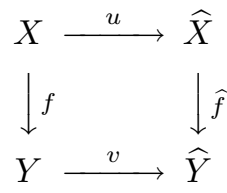

where $u$ and $v$ are trivial cofibrations and $\widehat{f}$ is a fibration of fibrant objects. Let $f^{\prime}: X^{\prime} \rightarrow Y$ be the pullback of $\widehat{f}$ along $v$, and note that the maps $X \rightarrow X^{\prime} \rightarrow \widehat{X}$ are weak equivalences since $\mathcal{C}$ is right proper. Factor $X \rightarrow X^{\prime}$ as the composite of a trivial cofibration $i: X \rightarrow X^{\prime \prime}$ and a trivial fibration $j: X^{\prime \prime} \rightarrow X^{\prime}$. Then the fibration $f: X \rightarrow Y$ is a retract of $j f^{\prime}: X^{\prime \prime} \rightarrow Y$ by the lifting axiom. Since $T$ preserves the fibrations $j$ and $f^{\prime}$, it also preserves $f$.

Lemma 10.6. For a $P_{W}$-cospectrum $A$, the functors $\theta_{A}: \mathcal{S} p \rightarrow \mathcal{S S}_{*}^{P_{W}}$ and $\phi_{A}$ : $\mathcal{S S}_{*}^{P_{W}} \rightarrow \mathcal{S} p$ are pointed simplicial left and right Quillen functors.

Proof. It suffices by Lemma 10.5 to show that $\phi_{A}$ preserves trivial fibrations and fibrations of fibrant objects. If $f: X \rightarrow Y$ is a trivial fibration in $\mathcal{S S}_{*}^{P_{W}}$ (and hence in $\mathcal{S S}_{*}$ ), then $\phi_{A}(f): \phi_{A}(X) \rightarrow \phi_{A}(Y)$ is a levelwise trivial fibration of spectra, and hence is a trivial fibration in $\mathcal{S} p$. If $f: X \rightarrow Y$ is a fibration of fibrant objects in $\mathcal{S S}_{*}^{P_{W}}$, then $\phi_{A}(f): \phi_{A}(X) \rightarrow \phi_{A}(Y)$ is a levelwise fibration of omega spectra, and hence is a fibration in $\mathcal{S} p$.

We let $\Theta_{A}: H o^{s} \rightarrow \mathcal{P}_{W}$ and $\Phi_{A}: \mathcal{P}_{W} \rightarrow H o^{s}$ denote the total left and right derived functors $\mathbf{L} \theta_{A}: H o(\mathcal{S} p) \rightarrow H o\left(\mathcal{S S}_{*}^{P_{W}}\right)$ and $\mathbf{R} \phi_{A}: H o\left(\mathcal{S S}_{*}^{P_{W}}\right) \rightarrow H o(\mathcal{S} p)$. Combining Lemma 10.6 with 10.1, we have

Theorem 10.7. For a $P_{W}$-cospectrum $A$, the functor $\Theta_{A}: H o^{s} \rightarrow \mathcal{P}_{W}$ is left adjoint to $\Phi_{A}: \mathcal{P}_{W} \rightarrow H o^{s}$ with $\operatorname{map}_{*}\left(\Theta_{A} E, X\right) \simeq \operatorname{map}_{*}\left(E, \Phi_{A} X\right)$ for $E \in H o^{s}$ and $X \in \mathcal{P}_{W}$. Moreover, there are natural equivalences $\Theta_{A}(K \wedge E) \simeq P_{W}\left(K \wedge \Theta_{A} E\right)$ and $\Phi_{A}\left(X^{K}\right) \simeq\left(\Phi_{A} X\right)^{K}$ for $K \in H o_{*}, E \in H o^{s}$, and $X \in \mathcal{P}_{W}$. Furthermore, the functor $\Phi_{A}: \mathcal{P}_{W} \rightarrow H o^{s}$ preserves homotopy fiber squares, while $\Theta_{A}: H o^{s} \rightarrow \mathcal{P}_{W}$ carries homotopy cofiber squares to $P_{W}$-homotopy cofiber squares.

10.8. The simplicial functoriality of $\Theta_{A}$ and $\Phi_{A}$. The total derived functors $\Theta_{A}=\mathbf{L} \theta_{A}: H o(\mathcal{S} p) \rightarrow H o\left(\mathcal{S S}_{*}^{P_{W}}\right)$ and $\Phi_{A}=\mathbf{R} \phi_{A}: H o\left(\mathcal{S S}_{*}^{P_{W}}\right) \rightarrow H o(\mathcal{S} p)$ are induced by simplicial functors $\Theta_{A}=\theta_{A} I: \mathcal{S} p \rightarrow \mathcal{S S}_{*}^{P_{W}}$ and $\Phi_{A}=\phi_{A} J: \mathcal{S S}_{*}^{P_{W}} \rightarrow$ $\mathcal{S} p$, which preserve weak equivalences, where $I: \mathcal{S} p \rightarrow \mathcal{S} p$ is a simplicial cofibrant replacement functor giving a cofibrant spectrum $I(E)$ with a natural trivial fibration $I(E) \rightarrow E$ for $E \in \mathcal{S} p$, and where $J: \mathcal{S S}_{*}^{P_{W}} \rightarrow \mathcal{S S}_{*}^{P_{W}}$ is a simplicial fibrant replacement functor giving a fibrant (i.e. $W$-null Kan) complex $J(X) \in \mathcal{S S}_{*}^{P_{W}}$ with a natural trivial cofibration (i.e. monic $P_{W}$-equivalence) $X \rightarrow J(X)$ for $X \in$ $\mathcal{S S}_{*}^{P_{W}}$. The functor $\Theta_{A}: \mathcal{S} p \rightarrow \mathcal{S S}_{*}^{P_{W}}$ preserves homotopy cofiber squares, while $\Phi_{A}: \mathcal{S S}_{*}^{P_{W}} \rightarrow \mathcal{S} p$ preserves homotopy fiber squares.

\section{The general FunCtors $\Phi_{A}$ And $\Theta_{A}$}

Extending the preceding work, we now introduce a general Kuhn functor $\Phi_{A}$ : $\mathcal{P}_{W} \rightarrow H o^{s}$ and its left adjoint $\Theta_{A}: H o^{s} \rightarrow \mathcal{P}_{W}$ for a "spectral $P_{W}$-cospectrum" $A$, where $W$ is a fixed pointed space. We first discuss 
11.1. Spectral $P_{W}$-cospectra. A spectral $P_{W}$-cospectrum $A$ consists of $P_{W^{-}}$ cospectra $A_{n}$ for $n \geq 0$ with structure maps $\sigma: S^{1} \wedge A_{n} \rightarrow A_{n+1}$ which are levelwise cofibrations of cospectra (in the sense that $\sigma: S^{1} \wedge A_{n}^{k} \rightarrow A_{n+1}^{k}$ is a cofibration in $\mathcal{S S}_{*}$ for $k, n \geq 0$ ).

11.2. The functor $\bar{\phi}_{A}: \mathcal{S S}_{*}^{P_{W}} \rightarrow \mathcal{C} s p \mathcal{S} p$. A cospectrum of spectra $E$ consists of spectra $E^{n}$ for $n \geq 0$ with structure maps $\sigma: E^{n+1} \wedge S^{1} \rightarrow E^{n}$. There is a proper pointed simplicial model category $\mathcal{C} s p \mathcal{S} p$ of cospectra of spectra, where a map $f: E \rightarrow F$ in $\mathcal{C} s p \mathcal{S} p$ is a weak equivalence (resp. cofibration) when $f$ induces weak equivalences (resp. cofibrations) $E^{n} \rightarrow F^{n}$ in $\mathcal{S} p$ for $n \geq 0$, and a fibration (resp. trivial fibration) when $f$ induces fibrations (resp. trivial fibrations) $E^{0} \rightarrow F^{0}$ and

$$
E^{n+1} \rightarrow F^{n+1} \times_{\left(F^{n}\right)^{S^{1}}}\left(E^{n}\right)^{S^{1}}
$$

in $\mathcal{S} p$ for $n \geq 0$. For a spectral $P_{W}$-cospectrum $A$, there is a pointed simplicial functor $\bar{\phi}_{A}: \mathcal{S S}_{*}^{P_{W}} \rightarrow \mathcal{C} \operatorname{spS} p$ where $\bar{\phi}_{A}(X)$ is the cospectrum of spectra with $\bar{\phi}_{A}(X)^{n}=\phi_{A_{n}}(X)$ for $n \geq 0$ and with the induced structure maps. Thus $\bar{\phi}_{A}(X)_{m}^{n}=\operatorname{map}_{*}\left(A_{n}^{m}, X\right)$ for $m, n \geq 0$, and $\bar{\phi}_{A}$ has a pointed simplicial left adjoint $\bar{\theta}_{A}: \mathcal{C} s p \mathcal{S} p \rightarrow \mathcal{S S}_{*}^{P_{W}}$ carrying a cospectrum of spectra $E$ to the pointed space $\bar{\theta}_{A}(E)$, which may be constructed as the quotient of $\bigvee_{m, n \geq 0}\left(A_{n}^{m} \wedge E_{m}^{n}\right)$ by the relations given by

$$
\begin{aligned}
& A_{n}^{m+1} \wedge E_{m+1}^{n} \stackrel{1 \wedge \sigma}{\longleftarrow} A_{n}^{m+1} \wedge S^{1} \wedge E_{m}^{n} \stackrel{\sigma \wedge 1}{\longrightarrow} A_{n}^{m} \wedge E_{m}^{n} \\
& A_{n+1}^{m} \wedge E_{m}^{n+1} \stackrel{\tau(1 \wedge \sigma)}{\longleftarrow} E_{m}^{n+1} \wedge S^{1} \wedge A_{n}^{m} \stackrel{\tau(\sigma \wedge 1)}{\longrightarrow} A_{n}^{m} \wedge E_{m}^{n}
\end{aligned}
$$

for $m, n \geq 0$ where $\tau$ is the twisting map.

Lemma 11.3. For a spectral $P_{W}$-cospectrum $A$, the functors $\bar{\theta}_{A}: \mathcal{C} s p \mathcal{S} p \rightarrow \mathcal{S S}_{*}^{P_{W}}$ and $\bar{\phi}_{A}: \mathcal{S S}_{*}^{P_{W}} \rightarrow \mathcal{C} \operatorname{sp} \mathcal{S} p$ are pointed simplicial left and right Quillen functors.

Proof. By Lemma 10.5, it suffices to show that $\bar{\phi}_{A}(f)$ is a trivial fibration (resp. fibration) in $\mathcal{C} s p \mathcal{S} p$ whenever $f: X \rightarrow Y$ is a trivial fibration (resp. fibration of fibrant objects) in $\mathcal{S S}_{*}^{P_{W}}$. This follows since the induced maps $\phi_{A_{0}}(X) \rightarrow \phi_{A_{0}}(Y)$ and

$$
\phi_{A_{n+1}}(X) \rightarrow \phi_{A_{n+1}}(Y) \times_{\phi_{A_{n}}(Y)^{S^{1}}} \phi_{A_{n}}(X)^{S^{1}}
$$

are levelwise trivial fibrations of spectra (resp. levelwise fibrations of omega spectra), and thus are trivial fibrations (resp. fibrations) in $\mathcal{C} s p \mathcal{S}$.

11.4. The functors $\beta: \mathcal{C} s p \mathcal{S} p \rightarrow \mathcal{T}$ owS $p$. A tower of spectra $E=\left\{E^{n}\right\}_{n \geq 0}$ consists of spectra $E^{n}$ with maps $E^{n+1} \longrightarrow E^{n}$ for $n \geq 0$. There is a proper pointed simplicial model category $\mathcal{T}$ ow $\mathcal{S} p$ of towers of spectra, where a map $f$ : $E \rightarrow F$ in $\mathcal{T}$ ow $\mathcal{S} p$ is a weak equivalence (resp. cofibration) when $f$ induces weak equivalences (resp. cofibrations) $E^{n} \rightarrow F^{n}$ in $\mathcal{S} p$ for $n \geq 0$, and a fibration (resp. trivial fibration) when $f$ induces fibrations (resp. trivial fibrations) $E^{0} \longrightarrow F^{0}$ and $E^{n+1} \longrightarrow F^{n+1} \times_{F^{n}} E^{n}$ in $\mathcal{S} p$ for $n \geq 0$. There is a pointed simplicial functor $\beta: \mathcal{C} s p \mathcal{S} p \longrightarrow \mathcal{T}$ ow $\mathcal{S} p$ where $\beta(X)$ is the tower of spectra with $\beta(X)^{n}=X^{n} \wedge S^{n}$ for $n \geq 0$. The functor $\beta$ has a pointed simplicial right adjoint $\gamma: \mathcal{T}$ ow $\mathcal{S} p \longrightarrow \mathcal{C} s p \mathcal{S} p$ where $\gamma(Y)$ is the cospectrum of spectra with $\gamma(Y)^{n}=\left(Y^{n}\right)^{S^{n}}$ for $n \geq 0$. It is now straightforward to prove the following. 
Lemma 11.5. The functors $\beta: \mathcal{C} s p \mathcal{S} p \longrightarrow \mathcal{T}$ ow $\mathcal{S} p$ and $\gamma: \mathcal{T}$ ow $\mathcal{S} p \longrightarrow \mathcal{C} s p \mathcal{S} p$ are pointed simplicial left and right Quillen equivalences.

Lemma 11.6. The constant tower functor $c^{*}: \mathcal{S} p \longrightarrow \mathcal{T}$ ow $\mathcal{S} p$ and the inverse limit functor $\lim : \mathcal{T}$ owS $p \longrightarrow \mathcal{S} p$ are pointed simplicial left and right Quillen functors.

Finally, for a spectral $P_{W}$-cospectrum $A$, we let $\Theta_{A}: H o^{s} \longrightarrow \mathcal{P}_{W}$ and $\Phi_{A}$ : $\mathcal{P}_{W} \longrightarrow H o_{*}^{s}$ denote the compositions of total derived functors

$$
\begin{aligned}
& H o(\mathcal{S} p) \stackrel{\mathbf{L} c^{*}}{\longrightarrow} H o(\mathcal{T} \text { ow } \mathcal{S} p) \stackrel{\mathbf{R} \gamma}{\longrightarrow} H o(\mathcal{C} s p \mathcal{S} p) \stackrel{\mathbf{L} \bar{\theta}_{A}}{\longrightarrow} H o\left(\mathcal{S S}_{*}^{P_{W}}\right) \\
& H o(\mathcal{S} p) \stackrel{\mathbf{R} \lim }{\longleftarrow} H o(\mathcal{T} \text { ow } \mathcal{S} p) \stackrel{\mathbf{L} \beta}{\longleftarrow} H o(\mathcal{C} s p \mathcal{S} p) \stackrel{\mathbf{R} \bar{\phi}_{A}}{\longleftarrow} H o\left(\mathcal{S S}_{*}^{P_{W}}\right)
\end{aligned}
$$

Noting that $\mathbf{R} \gamma$ and $\mathbf{L} \beta$ are adjoint equivalences, we obtain

Theorem 11.7. Theorem 10.7 remains valid when "a $P_{W}$-cospectrum $A$ " is replaced by "a spectral $P_{W}$-cospectrum $A . "$

11.8. The simplicial functoriality of $\Phi_{A}$ and $\Theta_{A}$. In each of the model categories $\mathcal{S S}_{*}^{P_{W}}, \mathcal{S} p, \mathcal{C} s p \mathcal{S} p$, and $\mathcal{T} o w \mathcal{S} p$, it is straightforward to construct a simplicial (co)fibrant replacement functor. Thus since $\Theta_{A}: H o(\mathcal{S} p) \longrightarrow H o\left(\mathcal{S S}_{*}^{P_{W}}\right)$ and $\Phi_{A}: H o\left(\mathcal{S S}_{*}^{P_{W}}\right) \longrightarrow H o(\mathcal{S} p)$ are composites of total derived functors of Quillen functors linking these categories, they are induced by simplicial functors $\Theta_{A}: \mathcal{S} p \longrightarrow \mathcal{S S}_{*}$ and $\Phi_{A}: \mathcal{S S}_{*}^{P_{W}} \longrightarrow \mathcal{S} p$ which preserve weak equivalences, as in 10.8. Moreover, the functor $\Theta_{A}: \mathcal{S} p \longrightarrow \mathcal{S S}_{*}^{P_{W}}$ preserves homotopy cofiber squares, while $\Phi_{A}: \mathcal{S S}_{*}^{P_{W}} \longrightarrow \mathcal{S} p$ preserves homotopy fiber squares.

Finally, we establish a key property of $\Phi_{A}$.

Theorem 11.9. For a spectral $P_{W}$-cospectrum $A$ and a $\Sigma^{\infty} W$-trivial spectrum $E \in H o^{s}$, there is a natural equivalence $\Phi_{A}\left(\Omega^{\infty} E\right) \simeq F\left(A_{*}^{0}, E\right)$ in $H o^{s}$ where $A_{*}^{0}$ is the edge-spectrum of $A$.

This will be proved in 11.11 using

11.10. Bispectra. A bispectrum $X$ consists of spaces $X_{m, n} \in \mathcal{S S}_{*}$ for $m, n \geq 0$ together with structure maps $\sigma_{1}: S^{1} \wedge X_{m, n} \longrightarrow X_{m+1, n}$ and $\sigma_{2}: S^{1} \wedge X_{m, n} \longrightarrow$ $S_{m, n+1}$ such that the compositions

$$
\sigma_{1}\left(1 \wedge \sigma_{2}\right), \sigma_{2}\left(1 \wedge \sigma_{1}\right)(\tau \wedge 1): S^{1} \wedge S^{1} \wedge X_{m, n} \rightarrow X_{m+1, n+1}
$$

are equal for $m, n \geq 0$ where $\tau$ is the twisting map. A bispectrum $X$ is called fibrant when the spaces $X_{m, n}$ are fibrant and the structure maps $X_{m, n} \longrightarrow\left(X_{m+1, n}\right)^{S^{1}}$ and $X_{m, n} \longrightarrow\left(X_{m, n+1}\right)^{S^{1}}$ are weak equivalences for $m, n \geq 0$. If $X$ is a fibrant bispectrum, then the edge spectra $X_{0, *}$ and $X_{*, 0}$ are related by a chain of natural weak equivalences

$$
\begin{gathered}
X_{0, *} \longrightarrow \operatorname{Sing}\left|X_{0, *}\right| \longleftarrow \text { Sing Tel }\left|X_{0, *}\right| \longrightarrow \text { Sing } \operatorname{Tel}^{(2)}\left|X_{*, *}\right| \\
\longleftarrow \text { Sing Tel }\left|X_{*, 0}\right| \longrightarrow \operatorname{Sing}\left|X_{*, 0}\right| \longleftarrow X_{*, 0}
\end{gathered}
$$

in $\mathcal{S} p$, where Sing is the singular functor, $|-|$ is the geometric realization functor, Tel is the telescope functor of Adams [Ada, p. 171], and $\mathrm{Tel}^{(2)}$ is the double telescope functor of Adams [Ada, pp. 173-176]. More generally, if $X$ is a cospectrum of fibrant bispectra, then the edge objects $X_{0, *} \in \mathcal{C} s p \mathcal{S} p$ and $X_{*, 0} \in \mathcal{C} s p \mathcal{S} p$ are related by a chain of natural weak equivalences in $\mathcal{C} s p \mathcal{S} p$ as displayed above. 
11.11. Proof of Theorem 11.9. Let $\phi(A, E)$ be the cospectrum of bispectra with $\phi(A, E)_{m, n}^{q}=\operatorname{map}_{*}\left(A_{q}^{m}, E_{n}\right)$ for $m, n, q \geq 0$. We may assume that the spectrum $E \in \mathcal{S} p$ is fibrant and conclude that $\phi(A, E)$ is a cospectrum of fibrant bispectra. Thus by 11.10 there is a natural equivalence $\phi\left(A_{*}^{*}, E_{0}\right) \simeq \phi\left(A_{*}^{0}, E_{*}\right)$ in $H o(\mathcal{C} s p \mathcal{S} p)$ which may be written as $\bar{\phi}_{A}\left(\Omega^{\infty} E\right) \simeq \phi\left(A_{*}^{0}, E_{*}\right)$ since $E_{0}=\Omega^{\infty} E$. This is carried to a natural equivalence $\Phi_{A}\left(\Omega^{\infty} E\right) \simeq F\left(A_{*}^{0}, E\right)$ by the functors

$$
H o(\mathcal{C} s p \mathcal{S} p) \stackrel{\mathbf{L} \beta}{\longrightarrow} H o(\mathcal{T} o w \mathcal{S} p) \stackrel{\mathbf{R} \lim }{\longrightarrow} H o(\mathcal{S} p) .
$$

\section{The FUnCtors $\Phi_{n}$ AND $\Theta_{n}$}

In final preparation for the proofs of Theorems 5.3 and 5.4, we now construct the required functors $\Phi_{n}: H o_{*} \longrightarrow H o^{s}$ and $\Theta_{n}: H o^{s} \longrightarrow H o_{*}$, and establish some of their formal properties. Throughout this section, we let $n \geq 1$ be a fixed integer, and we use the nullification functor $L_{n}^{f}=P_{\breve{A}(n+1)}$. A spectral $L_{n}^{f}$-cospectrum $B$ will be called admissible if the edge spectrum $B_{*}^{0}$ is equivalent to $C_{n-1}^{f} S_{(p)}$ in $H o^{s}$ and if the spectra $\Sigma^{\infty} B_{i}^{j}$ are in $\mathcal{F}_{n}$ for $i, j \geq 0$.

Theorem 12.1. For $n \geq 1$, there exists an admissible spectral $L_{n}^{f}$-cospectrum $B$.

Proof. As in 3.2, let $F(n)$ be a finite $p$-local spectrum of type $n$. The $F(n)$ trivialization of the $p$-local sphere spectrum $S_{(p)} \longrightarrow L_{n-1}^{f} S_{(p)}$ is given by the mapping telescope of a sequence $S_{(p)}=Y_{0} \rightarrow \cdots \rightarrow Y_{k} \rightarrow Y_{k+1} \rightarrow \cdots$ where the homotopy cofiber of $Y_{k} \rightarrow Y_{k+1}$ is a finite wedge of integral suspensions of $F(n)$. Thus, since $C_{n-1}^{f} S_{(p)}$ is the homotopy fiber of $S_{(p)} \rightarrow L_{n-1}^{f} S_{(p)}$, it is given by the mapping telescope of a sequence $X_{0} \stackrel{u_{0}}{\longrightarrow} X_{1} \rightarrow \cdots \rightarrow X_{i} \stackrel{u_{i}}{\longrightarrow} X_{i+1} \rightarrow \cdots$ of spectra in $\mathcal{F}_{n}$. Using the periodicity theorem of Hopkins and Smith (see 3.5), we may choose $v_{n}$-maps $\omega_{i}: X_{i} \wedge S^{d_{i}} \rightarrow X_{i}$ in $H o^{s}$ with $d_{i} \mid d_{i+1}$ and with $\omega_{i+1} u_{i}=u_{i}\left(\omega_{i}\right)^{q_{i}}$ for $i \geq 0$ and $q_{i}=d_{i+1} / d_{i}$. We may then desuspend these spectra and maps to give: spaces $\widetilde{X}_{i} \in H o_{*}$ for $i \geq 0$ with $\Sigma^{\infty} \widetilde{X}_{i}=S^{r_{i}} \wedge X_{i}$ where $r_{i+1} \geq r_{i} \geq 0$; maps $\widetilde{\omega}_{i}: \widetilde{X}_{i} \wedge S^{d_{i}} \rightarrow \widetilde{X}_{i}$ in $H o_{*}$ for $i \geq 0$ which are equivalent to double suspensions with $\Sigma^{\infty} \widetilde{\omega}_{i}=S^{r_{i}} \wedge \omega_{i}$; and maps $\widetilde{u}_{i}: S^{r_{i+1}-r_{i}} \wedge \widetilde{X}_{i} \rightarrow \widetilde{X}_{i+1}$ in $H o_{*}$ for $i \geq 0$ with $\Sigma^{\infty} \widetilde{u}_{i}=S^{r_{i+1}} \wedge u_{i}$ and with

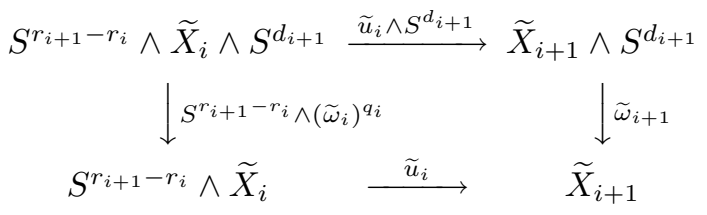

commuting in $H o_{*}$. The maps $\widetilde{\omega}_{i}: \widetilde{X}_{i} \wedge S^{d_{i}} \rightarrow \widetilde{X}_{i}$ are $L_{n}^{f}$-equivalences by Bou7. 11.12-11.14]. We next rigidify this homotopy system by choosing a representative sequence of fibrant spaces $\widetilde{X}_{i}$ and cofibrations $\widetilde{u}_{i}: S^{r_{i+1}-r_{i}} \wedge \widetilde{X}_{i} \rightarrow \widetilde{X}_{i+1}$ in $\mathcal{S S}_{*}$, and then choosing a representative sequence of maps $\widetilde{\omega}_{i+1}: \widetilde{X}_{i+1} \wedge S^{d_{i+1}} \rightarrow \widetilde{X}_{i+1}$ in $\mathcal{S S}_{*}$ making the above-displayed square commute for each $i$. For $i \geq 0$ and $m \geq d_{i}$ with $d_{i} \mid m$, we let $B\left(\widetilde{X}_{i}, \widetilde{\omega}_{i}, m\right)$ be the obvious $L_{n}^{f}$-cospectrum with $B\left(\widetilde{X}_{i}, \widetilde{\omega}_{i}, m\right)^{k}=$ $\widetilde{X}_{i} \wedge S^{r}$ for $k \equiv-r \bmod m$ and $0 \leq r<m$. We inductively construct $L_{n}^{f}$-cospectra $B\left(\tilde{X}_{i}\right)$ for $i \geq 0$, together with maps $B\left(\widetilde{X}_{i}, \widetilde{\omega}_{i}, d_{i}\right) \rightarrow B\left(\tilde{X}_{i}\right)$ and $S^{r_{i+1}-r_{i}} \wedge$ 
$B\left(\widetilde{X}_{i}\right) \rightarrow B\left(\widetilde{X}_{i+1}\right)$, by taking the pushouts

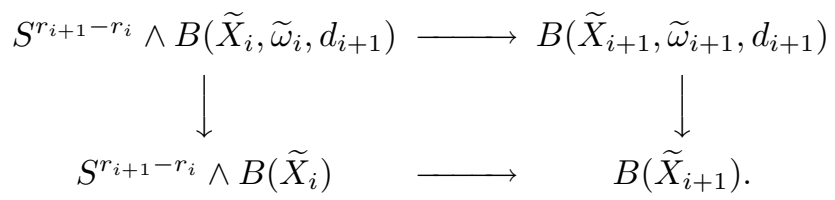

We note that $B\left(\widetilde{X}_{i}\right)^{0}=\widetilde{X}_{i}$ for each $i \geq 0$. Finally, we let $B$ be the associated spectral $L_{n}^{f}$-cospectrum with $B_{r_{i}+j}^{*}=S^{j} \wedge B\left(\widetilde{X}_{i}\right)$ for $0 \leq j<r_{i+1}-r_{i}$.

12.2. The functors $\Phi_{n}$ and $\Theta_{n}$. Holding $n \geq 1$ fixed, we choose an admissible spectral $L_{n}^{f}$-cospectrum $B$, together with an equivalence $B_{*}^{0} \simeq C_{n-1}^{f} S_{(p)}$ and obtain the adjoint functors $\Theta_{B}: H o^{s} \rightarrow \mathcal{U} \mathcal{L}_{n}^{f}$ and $\Phi_{B}: \mathcal{U} \mathcal{L}_{n}^{f} \rightarrow H o^{s}$. Using the reflection $L_{n}^{f}: H o_{*} \longrightarrow \mathcal{U} \mathcal{L}_{n}^{f} \subset H o_{*}$, we extend these functors to give $\Theta_{n}=\Theta_{B}: H o^{s} \longrightarrow$ $H o_{*}$ and $\Phi_{n}=\Phi_{B} L_{n}^{f}: H o_{*} \longrightarrow H o^{s}$. We now have

Theorem 12.3. For $n \geq 1$, the functors $\Phi_{n}: H o_{*} \longrightarrow H o^{s}$ and $\Theta_{n}: H o^{s} \rightarrow H o_{*}$ satisfy the conditions of Theorem 5.3(iv), (v) and Theorem 5.4(ii), (iv), (v).

Proof. Condition 5.3(v) follows from Lemma 12.6 below, while the other conditions follow from Theorem 11.7.

Condition 5.3(v) requires additional work to show that $\Phi_{n}: H o_{*} \rightarrow H o^{s}$ preserves homotopy fiber squares in $H o_{*}$, not merely $\mathcal{U L}_{n}^{f}$.

Lemma 12.4. If $f: X \rightarrow Y$ is a map in $\mathcal{U} \mathcal{L}_{n}^{f}$ with $f_{*}: \pi_{i} X \cong \pi_{i} Y$ for sufficiently large $i$, then $\Phi_{B} f: \Phi_{B} X \simeq \Phi_{B} Y$.

Proof. It suffices to show that $\operatorname{map}_{*}\left(B_{m}^{k}, f\right)$ is an equivalence for $k, m \geq 0$. This follows since $\operatorname{map}_{*}\left(B_{m}^{k+j} \wedge S^{j}, f\right)$ is an equivalence for sufficiently large $j$ and since there is an $L_{n}^{f}$-equivalence $B_{m}^{k+j} \wedge S^{j} \rightarrow B_{m}^{k}$ for all $j$.

Lemma 12.5. For a homotopy fiber sequence $F \rightarrow X \rightarrow Y$ of pointed spaces and $n \geq 0$, the map $L_{n}^{f} F \rightarrow \operatorname{fib}\left(L_{n}^{f} X \rightarrow L_{n}^{f} Y\right)$ has homotopy fiber with vanishing homotopy groups in dimensions $\geq d_{n}$.

Proof. Since $L_{n}^{f} X \simeq P_{(p)} P_{A(n+1)} X$ by Lemma 4.10, this follows from [Bou7 8.1].

Lemma 12.6. The functor $\Phi_{n}: H o_{*} \rightarrow H o^{s}$ preserves homotopy fiber squares.

Proof. For a homotopy pullback $X \times_{Y} Z$, the map $L_{n}^{f}\left(X \times_{Y} Z\right) \rightarrow L_{n}^{f} X \times_{L_{n}^{f} Y} L_{n}^{f} Z$ is a $\pi_{i}$-equivalence for $i>d_{n}+1$ by Lemma 12.5 , and is consequently a $\Phi_{B}$-equivalence by Lemma 12.4. The result now follows since $\Phi_{B}$ preserves homotopy fiber squares in $\mathcal{U} \mathcal{L}_{n}^{f}$ by Theorem 11.7 .

12.7. The simplicial functoriality of $\Phi_{n}$ and $\Theta_{n}$. The functors $\Phi_{n}: H o_{*} \rightarrow$ $H o^{s}$ and $\Theta_{n}: H o^{s} \rightarrow H o_{*}$ for $n \geq 1$ are induced by simplicial functors

$$
\begin{aligned}
& \Phi_{n}=\Phi_{B} L_{n}^{f}: \mathcal{S S}_{*} \longrightarrow \mathcal{S} p \\
& \Theta_{n}=L_{n}^{f} \Theta_{B}: \mathcal{S}_{p} \longrightarrow \mathcal{S S}_{*}
\end{aligned}
$$

which are constructed using the simplicial functors $\Phi_{B}, \Theta_{B}$, and $L_{n}^{f}$ of 11.8 and 4.4. By 11.8 and the proof of Lemma 12.6, $\Phi_{n}: \mathcal{S S}_{*} \longrightarrow \mathcal{S} p$ preserves homotopy fiber squares, while $\Theta_{n}: \mathcal{S} p \longrightarrow \mathcal{S S}_{*}$ carries homotopy cofiber squares to $L_{n}^{f}$-homotopy cofiber squares. 


\section{Proofs of Theorems 5.3 And 5.4}

We now proceed to prove Theorems 5.3 and 5.4 by establishing the remaining properties of $\Phi_{n}$ and $\Theta_{n}$ not covered by Theorem 12.3. As above, we hold $n \geq 1$ fixed and let $B$ be an admissible spectral $L_{n}^{f}$-cospectrum with a given equivalence $B_{*}^{0} \simeq C_{n-1}^{f} S_{(p)}$ in $H o^{s}$.

Theorem 13.1. For $n \geq 1$ and $E \in H o^{s}$, there is a natural equivalence $\Phi_{n} \Omega^{\infty} E \simeq$ $\widehat{M}_{n}^{f} E$ in $H o^{s}$.

Proof. Since the map $L_{n}^{f} \Omega^{\infty} E \rightarrow \Omega^{\infty} L_{n}^{f} E$ is a $\pi_{i}$-equivalence for $i>d_{n}$ by Proposition 5.2, there are natural equivalences

$$
\begin{aligned}
\Phi_{n} \Omega^{\infty} E & \simeq \Phi_{B} L_{n}^{f} \Omega^{\infty} E \simeq \Phi_{B} \Omega^{\infty} L_{n}^{f} E \\
& \simeq F\left(B_{*}^{0}, L_{n}^{f} E\right) \simeq F\left(C_{n-1}^{f} S_{(p)}, L_{n}^{f} E\right) \simeq \widehat{M}_{n}^{f} E
\end{aligned}
$$

by Lemma 12.4, Theorem 11.9, and Theorem 3.3.

Theorem 13.2. For $n \geq 1$, let $A$ be an $L_{n}^{f}$-cospectrum with $\Sigma^{\infty} A^{i} \in \mathcal{F}_{n}$ for $i \geq 0$. Then for each space $X \in \mathcal{U} \mathcal{L}_{n}^{f}$, the spectrum $\Phi_{A} X$ belongs to $\mathcal{M}_{n}^{f} \cap \widehat{\mathcal{M}}_{n}^{f}$.

Proof. $\Phi_{A} X$ belongs to $\mathcal{L}_{n}^{f}$ since it is an $\Omega$-spectrum whose spaces $X^{A^{i}}$ belong to $\mathcal{U} \mathcal{L}_{n}^{f}$ for $i \geq 0$. Moreover,

$$
v_{k}^{-1} \pi_{*}\left(\Phi_{A} X ; F(k)\right) \cong v_{k}^{-1} \pi_{*}\left(X^{A^{0}} ; F(k)\right) \cong v_{k}^{-1} \pi_{*}\left(X ; A^{0} \wedge F(k)\right)=0
$$

for $1 \leq k<n$ since $A^{0} \wedge F(k) \in \mathcal{F}_{n}$, and $\pi_{*} \Phi_{A} X$ is $p$-torsion since $\left[\Sigma^{j} A^{i}, \Sigma^{j} A^{i}\right]$ is a finite $p$-torsion group for $i \geq 0$ and $j \geq 2$. Hence, $L_{n-1}^{f} \Phi_{A} X=0$ by 3.8, and $\Phi_{A} X$ belongs to $\mathcal{M}_{n}^{f}$. For $1 \leq k<n$ choose a map $\omega_{k}: \Sigma^{d} W_{k} \rightarrow W_{k}$ of pointed spaces such that $\omega_{k} \wedge A^{0}$ is trivial and $\Sigma^{\infty} \omega_{k}$ is a $v_{k}$-map of finite $p$-local spectra of type $k$, and also let $\omega_{0}: \Sigma^{d} W_{0} \rightarrow W_{0}$ denote a map $S_{(p)}^{m} \rightarrow S_{(p)}^{m}$ of degree $p^{j}$ for some $m, j>0$ such that $\omega_{0} \wedge A^{0}$ is trivial. Let $\omega_{k}^{\#}:\left(\Phi_{A} X\right)^{W_{k}} \rightarrow\left(\Phi_{A} X\right)^{\Sigma^{d} W_{k}}$ be the induced map in $\mathcal{M}_{n}^{f}$ for $0 \leq k<n$. Then $\widehat{M}_{n}^{f} \omega_{k}^{\#}$ is trivial since $\widehat{M}_{n}^{f} \omega_{k}^{\#}=$ $\Phi_{n} \Omega^{\infty} \omega_{k}^{\#}$ by Theorem 13.1 where $\Omega^{\infty} \omega_{k}^{\#}$ is trivial. Thus $\omega_{k}^{\#}$ is trivial by Theorem 3.3, and $\Phi_{A} X$ is $F(n)_{*}$-local by [Bou8, 6.6]. Hence $\Phi_{A} X$ belongs to $\widehat{\mathcal{M}}_{n}^{f}$ since $\widehat{M}_{n}^{f}=L_{F(n)} L_{n}^{f}$ by Theorem 3.3 .

Theorem 13.3. For $n \geq 1$ and $X \in H o_{*}$, the spectrum $\Phi_{n} X$ belongs to $\widehat{\mathcal{M}}_{n}^{f}$.

Proof. This follows since $\Phi_{n} X$ is a homotopy inverse limit of spectra $S^{m} \wedge \Phi_{B_{m}^{*}} L_{n}^{f} X$ which are in $\widehat{\mathcal{M}}_{n}^{f}$ by Theorem 13.2.

Theorem 13.4. For $n \geq 1$ and $W \in \mathcal{F}_{n}$, there are isomorphisms

$$
v_{n}^{-1} \pi_{*}(X ; W) \cong\left[W, \Phi_{n} X\right]_{*} \cong v_{n}^{-1} \pi_{*}\left(\Phi_{n} X ; W\right)
$$

which are natural in $X \in H o_{*}$.

Proof. By desuspending a $v_{n}$-map for $W$, we may obtain an $L_{n}^{f}$-cospectrum $A$ such that $\Sigma^{\infty} A^{i} \in \mathcal{F}_{n}$ for $i \geq 0, \Sigma^{\infty} A^{0} \simeq \Sigma^{j} W$ for some $j$, and $\pi_{*} \Phi_{A} X \cong v_{n}^{-1} \pi_{*}\left(X ; A^{0}\right)$ naturally for $X \in \mathcal{U L}_{n}^{f}$. Then for $X \in H o_{*}$ there are natural equivalences

$$
\Phi_{A} L_{n}^{f} X \simeq \widehat{M}_{n}^{f} \Phi_{A} L_{n}^{f} X \simeq \Phi_{n} \Omega^{\infty} \Phi_{A} L_{n}^{f} X \simeq \Phi_{n}\left(L_{n}^{f} X\right)^{A^{0}} \simeq\left(\Phi_{n} X\right)^{A^{0}}
$$


by Theorems 13.1, 13.2, and 12.3. Hence for $X \in H o_{*}$ there are natural isomorphisms $\pi_{*} \Phi_{A} L_{n}^{f} X \cong \pi_{*}\left(\Phi_{n} X\right)^{A^{0}}$ giving isomorphisms

$$
v_{n}^{-1} \pi_{*}\left(L_{n}^{f} X ; A^{0}\right) \cong\left[A^{0}, \Phi_{n} X\right]_{*},
$$

and the result follows by 4.5 and 4.6 .

Corollary 13.5. For $n \geq 1$, if $f: X \rightarrow Y$ is a $v_{n}^{-1} \pi_{*}$-equivalence in $H o_{*}$, then $\Phi_{n} f: \Phi_{n} X \simeq \Phi_{n} Y$.

Proof. Since $\Phi_{n} f$ is a $v_{n}^{-1} \pi_{*}$-equivalence in $\widehat{\mathcal{M}}_{n}^{f}$ by 13.3 and 13.4 , it is an equivalence by 3.8 .

Theorem 13.6. For $n \geq 1$ and $E \in H o^{s}, \Theta_{n} E$ belongs to $\mathcal{U} \mathcal{N}_{n}^{f}$.

Proof. The maps $L_{n-1}^{f} \Theta_{n} E \rightarrow *$ and $\left(\Theta_{n} E\right)\left\langle d_{n}\right\rangle \rightarrow \Theta_{n} E$ are $\Phi_{n}$-equivalences by Corollary 13.5, and thus are $\left[\Theta_{n} E,-\right]$-equivalences since $\Theta_{n}: H o^{s} \rightarrow \mathcal{U} \mathcal{L}_{n}^{f}$ is left adjoint to $\Phi_{n}: \mathcal{U} \mathcal{L}_{n}^{f} \rightarrow H o^{s}$ by Theorem 12.3 . Hence $\Theta_{n} E$ belongs to $\mathcal{U} \mathcal{M}_{n}^{f}$ since the localization map $\Theta_{n} E \rightarrow L_{n-1}^{f} \Theta_{n} E$ is trivial, and $\Theta_{n} E$ belongs to $\mathcal{U N}_{n}^{f}$ since its identity map factors through $\left(\Theta_{n} E\right)\left\langle d_{n}\right\rangle$.

Theorem 13.7. For $n \geq 1$ and $E \in H o^{s}$, there is a natural equivalence $L_{n}^{f} \Sigma^{\infty} \Theta_{n} E$ $\simeq M_{n}^{f} E$.

Proof. Since the functors

$$
H o^{s} \stackrel{\Theta_{n}}{\longrightarrow} \mathcal{U} \mathcal{L}_{n}^{f} \stackrel{L_{n}^{f} \Sigma^{\infty}}{\longrightarrow} \mathcal{L}_{n}^{f}
$$

are left adjoint to

$$
H o s \stackrel{\Phi_{n}}{\longleftarrow} \mathcal{U} \mathcal{L}_{n}^{f} \stackrel{\Omega^{\infty}}{\longleftarrow} \mathcal{L}_{n}^{f},
$$

and since the functors $M_{n}^{f}: H o^{s} \rightleftarrows \mathcal{L}_{n}^{f}: \widehat{M}_{n}^{f}$ are also adjoint, the natural equivalence $\Phi_{n} \Omega^{\infty} E \simeq \widehat{M}_{n}^{f} E$ of Theorem 13.1 implies a natural equivalence $L_{n}^{f} \Sigma^{\infty} \Theta_{n} E \simeq$ $M_{n}^{f} E$.

13.8. Proof of Theorem 5.3. Parts (i), (ii), and (iii) follow by Theorems 13.3, 13.4, and 13.1, while parts (iv) and (v) follow by Theorem 12.3.

13.9. Proof of Theorem 5.4. Parts (i) and (iii) follow by Theorems 13.6 and 13.7, while parts (ii), (iv), and (v) follow by Theorem 12.3 .

\section{REFERENCES}

[Ada] J. F. Adams, Stable Homotopy and Generalized Homology, University of Chicago Press, 1974. MR 53:6534

[Bor] F. Borceux, Handbook of Categorical Algebra 2, Categories and Structures, Encyclopedia of Mathematics and its Applications, vol. 51, Cambridge University Press, 1994. MR 96g:18001b

[Bou1] A.K. Bousfield, Boolean algebra of spectra, Comment. Math. Helv 54 (1979), 368-377; Correction, Comment Math. Helv. 58 (1983), 599-600. MR 81a:55015 MR 85h:55013

[Bou2] , The localization of spectra with respect to homology, Topology 18 (1979), 257281. MR 80m:55006

[Bou3] Cohomological localizations of spaces and spectra, preprint, 1979.

[Bou4],$K$-localizations and $K$-equivalences of infinite loop spaces, Proc. London Math. Soc. 44 (1982), 291-311. |MR 83g:55008

[Bou5] - On homology equivalences and homological localizations of spaces, Amer. J. Math. 104 (1982), 1025-1042. MR 84g:55014 
[Bou6] Uniqueness of infinite deloopings for $K$-theoretic spaces, Pacific J. Math. 129 (1987), 1-31. MR 89g:55017

[Bou7] Localization and periodicity in unstable homotopy theory, J. Amer. Math. Soc. 7 (1994), 831-873. MR 95c:55010

[Bou8] _ Unstable localization and periodicity, Algebraic Topology: New Trends in Localization and Periodicity, Progress in Mathematics, vol. 136, Birkhauser-Verlag, 1996, pp. 33-50. MR 98c:55014

[Bou9] _ Homotopical localizations of spaces, Amer. J. Math. 119 (1997), 1321-1354. MR 98m:55009

[Bou10] — On $K(n)$-equivalences of spaces, Homotopy invariant algebraic structures: a conference in honor of J. Michael Boardman, Contemp. Math., vol. 239. CMP 2000:03

[BF] A.K. Bousfield and E.M. Friedlander, Homotopy theory of $\Gamma$-spaces, spectra, and bisimplicial sets, Lecture Notes in Math., vol. 658, Springer-Verlag, 1978, pp. 80-130. MR 80e:55021

[BK] A.K. Bousfield and D.M. Kan, Homotopy Limits, Completions and Localizations, Lecture Notes in Math., vol. 304, Springer-Verlag, 1972. MR 51:1825

[Dro] E. Dror Farjoun, Cellular spaces, null spaces and homotopy localizations, Lecture Notes in Math., vol. 1622, Springer-Verlag, 1996. MR 98f:55010

[DDK] E. Dror Farjoun, W.G. Dwyer, and D.M. Kan, An arithmetic square for virtually nilpotent spaces, Illinois J. Math. 21 (1977), 242-254. MR 55:11246

[DS] W.G. Dwyer and J. Spalinski, Homotopy theories and model categories, Handbook of Algebraic Topology, North-Holland, 1995, pp. 73-126. MR 96h:55014

[GJ] P.G. Goerss and J.F. Jardine, Simplicial Homotopy Theory, Progress in Mathematics, vol. 174, Birkhauser-Verlag, 1999. CMP 2000:02

[Hir] P.S. Hirschhorn, Localization in Model Categories, in preparation.

[Hop] M.J. Hopkins, Global methods in homotopy theory, Homotopy Theory - Proceedings of the Durham Symposium 1985, London Math. Soc. Lecture Note Series, vol. 117, Cambridge Univ. Press, 1987, pp. 73-96. MR 89g:55022

[HRW] M.J. Hopkins, D.C. Ravenel, and W.S. Wilson, Morava Hopf algebras and spaces K( $n)$ equivalent to finite Postnikov systems, Stable and Unstable Homotopy, Fields Institute Communications, vol. 19, 1998, pp. 137-163. MR 99e:55005

[HSm] M.J. Hopkins and J.H. Smith, Nilpotence and stable homotopy theory II, Ann. Math. 148 (1998), 1-49. MR 99h:55009

[Hov] M. Hovey, Model Categories, Mathematical Surveys and Monographs, vol. 63, American Mathematical Society, 1998. MR 99h:55031

[HP] M. Hovey and J.H. Palmieri, The structure of the Bousfield lattice, Homotopy invariant algebraic structures: a conference in honor of J. Michael Boardman, Contemp. Math., vol. 239. CMP 2000:03

[HPS] M. Hovey, J.H. Palmieri, and N.P. Strickland, Axiomatic Stable Homotopy Theory, Mem. Amer. Math. Soc. (1997). MR 98a:55017

[HSS] M. Hovey, B. Shipley, and J.H. Smith, Symmetric spectra, J. Amer. Math. Soc. 13 (2000), 149-208. CMP 2000:02

[HSt] M. Hovey and N.P. Strickland, Morava K-theories and localization, Mem. Amer. Math. Soc. 139 (1999). MR 99b:55017

[Kas] T. Kashiwabara, On Brown-Peterson cohomology of $Q X$ (to appear).

[Kuh1] N.J. Kuhn, Suspension spectra and homology equivalences, Trans. Amer. Math. Soc. 283 (1984), 303-313. MR 85g:55014

[Kuh2] _ Morava K-theories and infinite loop spaces, Lecture Notes in Math., vol. 1370, Springer-Verlag, 1989, pp. 243-257. MR 90d:55014

[Mah] M.E. Mahowald, The image of $J$ in the EHP sequences, Ann. Math. 116 (1982), 65-112. MR 86d:55018

[MS] M. Mahowald and H. Sadofsky, $v_{n}$-telescopes and the Adams spectral sequence, Duke Math. J. 78 (1995), 101-129. MR 96h:55006

[Mil1] H.R. Miller, On relations between Adams spectral sequences with an application to stable homotopy theory, J. Pure Appl. Algebra 20 (1981), 287-312. MR 82f:55029

[Mil2] , Finite localizations, Bol. Soc. Mat. Mexicana (Homenaje a Jose Adem) 37 (1992), 383-390. MR 96h:55009 
[Qui] D.G. Quillen, Homotopical Algebra, Lecture Notes in Math., vol. 43, Springer-Verlag, 1967. MR 36:6480

[Rav1] D.C. Ravenel, Localization with respect to certain periodic homology theories, Amer. J. Math. 106 (1984), 351-414. MR 85k:55009

[Rav2] - Progress report on the telescope conjecture, London Math. Soc. Lecture Note Series 176, Adams Memorial Symposium on Algebraic Topology, Cambridge Univ. Press, 1992, pp. 1-21. MR 94h:55023

[Rav3] _ Nilpotence and Periodicity in Stable Homotopy Theory, Ann. of Math. Studies 128, Princeton Univ. Press, 1992. MR 94b:55015

[Rav4] , Life after the telescope conjecture, Algebraic $K$-Theory and Algebraic Topology (P.G. Goerss and J.F. Jardine, eds.), NATO Adv. Sci. Inst. Ser. C Math. Phys. Sci. 407, Kluwer, 1993, pp. 205-222. MR 96i:55016

[RW] D.C. Ravenel and W.S. Wilson, The Morava K-theories of Eilenberg-Mac Lane spaces and the Conner-Floyd conjecture, Amer. J. Math. 102 (1980), 691-748. MR 81i:55005

[War] R.B. Warfield, Jr., Nilpotent Groups, Lecture Notes in Math., vol. 513, Springer-Verlag, 1976. MR 53:13413

Department of Mathematics, Statistics, and Computer Science (M/C 249), University of Illinois at Chicago, Chicago, Illinois 60607

E-mail address: bous@uic.edu 